\title{
Synthesis of Multifunctional Aryl(trifloxyalkenyl)iodonium Triflate Salts
}

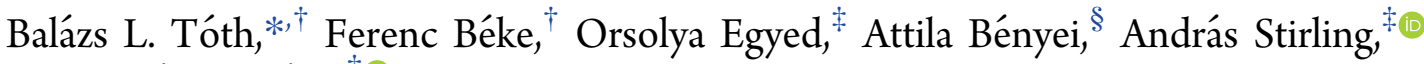 \\ and Zoltán Novák* ${ }^{*}+\odot$
}

\begin{abstract}
†ELTE "Lendület” Catalysis and Organic Synthesis Research Group, Institute of Chemistry, Eötvös Loránd University, Faculty of Science, Pázmány Péter stny. 1/A, H-1117 Budapest, Hungary

${ }^{\ddagger}$ Research Centre for Natural Sciences of the Hungarian Academy of Sciences, Magyar Tudósok körútja 2, H-1117 Budapest, Hungary

${ }^{\S}$ Department of Pharmaceutical Chemistry, University of Debrecen, Egyetem tér 1, H-4032 Debrecen, Hungary
\end{abstract}

\section{Supporting Information}

ABSTRACT: A convenient procedure for the synthesis of $\operatorname{aryl}($ trifloxyalkenyl)iodonium triflate salts from commercially available (diacetoxyiodo)benzene, trimethylsilyl trifluoromethanesulfonate, and acetylenes under mild conditions was developed. The obtained multifunctional hypervalent vinyliodonium salts equipped with electrophilic and nucleophilic functions could serve as novel C2 synthons for organic transformations. The structure of the iodonium salts was identified by multidimensional NMR spectroscopy and X-ray crystallography.

\section{INTRODUCTION}

In the last decade, the synthesis and application of hypervalent iodine reagents became a hot topic in synthetic organic chemistry. Due to their electrophilic character, high reactivity, general low toxicity, and easy preparation, +3 oxidation state organic iodonium salts are frequently used in arylation, alkenylation, alkynylation, or alkylation reactions. ${ }^{1}$ Although the number of applications of these hypervalent reagents increases, the synthesis and utilization of alkenyl derivatives ${ }^{2}$ are still a less explored topic of hypervalent iodine chemistry. ${ }^{3}$ However, the alkenyl function appears as an important molecular motif in innumerable syntheses of valuable chemical compounds. ${ }^{4}$ Therefore, the design of direct alkenylation reactions and multifunctional alkenyl building blocks has great synthetic potential. ${ }^{5}$ To fulfill this synthetic demand, (trifloxyalkenyl)iodonium triflate salts ${ }^{6}$ were designed and utilized in ligand exchange reactions resulting various diaryliodonium species, ${ }^{7}$ palladium-catalyzed cross-coupling reactions, ${ }^{8}$ and also in metal-free substituted oxazole ring formation reaction. ${ }^{9}$

Although the synthesis of aryl(trifloxyalkenyl)iodonium triflate salts can be achieved starting from iodosylbenzene, trifluoromethanesulfonic acid, and acetylenes, ${ }^{10}$ their synthesis is based on the utilization of less available $\mathrm{I}(\mathrm{III})$ species (prepared from DIB) and highly sensitive TfOH. Most importantly, the efficiency of the synthesis is limited to $50 \%$ maximum theoretical yield because of the $1: 1$ ratio of $\lambda^{3}$ iodane source to triflate anion (Scheme 1). Of note, there are three examples for the syntheses of the target alkenes, starting from ArI or $\mathrm{PhI}(\mathrm{OAc})_{2}$, but the first strategy uses special FXeOTf reagent, ${ }^{6 \mathrm{e}}$ and there are only two reactions of DIB with 2 equiv of TfOH and terminal alkyne, which reportedly reach 55 and $56 \%$ isolated yield, respectively. ${ }^{7 a, c}$

To overcome the limitations of the aforementioned existing procedures and expand the chemical space of available alkenyl(aryl)iodonium salts, we aimed to design new procedure and synthetic approach for the efficient synthesis of novel multifunctional alkenyl synthons using less corrosive trimethylsilyl triflate (TMSOTf) as triflate source and readily available aryliodonium source such as (diacetoxyiodo)benzene (DIB, 3) (Scheme 2). Our synthetic strategy is based on the utilization of both internal and terminal acetylenes containing versatile alkyl, aryl, and hetaryl fragments equipped with various synthetically useful functional groups $(\mathrm{OH}, \mathrm{Br}, \mathrm{Cl}$, $\mathrm{CN})$.

\section{RESULTS AND DISCUSSION}

We began the synthetic investigation with the preparation of phenyl(trifloxyalkenyl)iodonium triflate salt from DIB and phenylacetylene using TMSOTf as activator. Compared to the final, optimized reaction conditions, the following modifications resulted in lower than $41 \%$ yield: the concentration of DIB higher than $0.5 \mathrm{~mol} / \mathrm{L}$, less inert or polar solvents, higher or lower reaction temperatures, longer reaction time, and less equivalent of added acetylene or TMSOTf (Scheme 3 ).

Additionally, increasing the amount of phenylacetylene up to 1.5 equiv did not give the target product in higher yield. Drawing the lessons, we determined the best synthetic

Received: March 15, 2019

Accepted: March 26, 2019

Published: May 24, 2019 
Scheme 1. Previous Literature Procedures, Highlighting the Formation of Trifloxyalkenyl(aryl)iodonium Triflates from Two Hypervalent Iodine Reagent

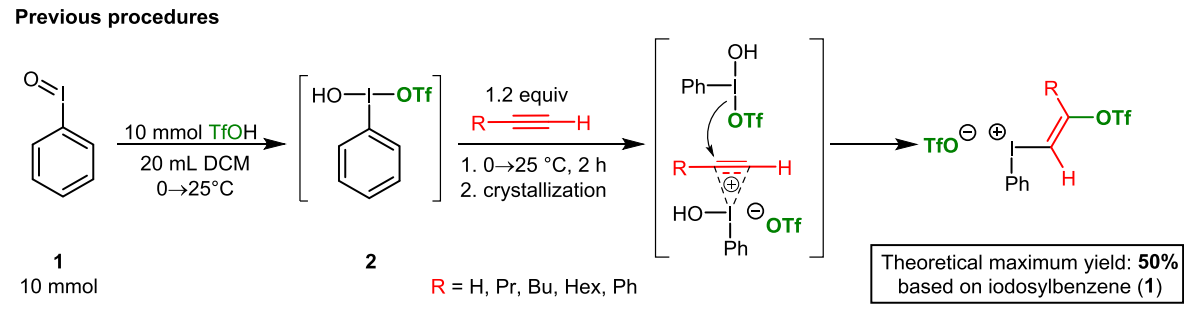

Scheme 2. Retrosynthetic Approach to the Access of Multifunctional Aryl(trifloxyalkenyl)iodonium Triflate Salts

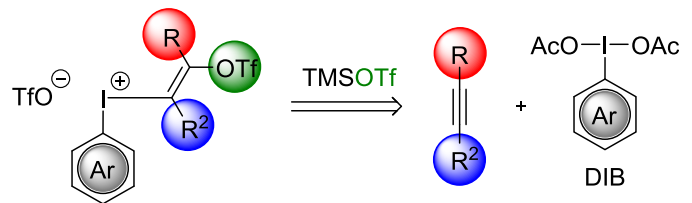

conditions to the access of the target compounds $6 \mathbf{a}$. To the anhydrous dichloromethane solution of DIB, 2 equiv of trimethylsilyl trifluoromethanesulfonate have been added at 0 ${ }^{\circ} \mathrm{C}$ and the temperature increased to $25{ }^{\circ} \mathrm{C}$. The colorless solution turns yellow by forming bis(trifloxy)iodobenzene (4). ${ }^{11}$ The solution was cooled again to $0{ }^{\circ} \mathrm{C}$, and the acetylene derivative was added dropwise. The reaction mixture darkened while the desired aryl(trifloxyalkenyl)iodonium triflate product forms and may precipitate from the solution at $0{ }^{\circ} \mathrm{C}$. For the completion of the reaction, we allowed to warm up the mixture to $25{ }^{\circ} \mathrm{C}$. As shown in Scheme 3, after the evaporation of solvent and recrystallization procedure, we obtained the unsubstituted phenyl derivative $6 \mathbf{a}$ as a white solid with $56 \%$ yield.

In general, the reaction took place in a similar manner, resulting in off-white solids after crystallization; nevertheless, the determination of their structure was a more complex task. To study the influence of steric and electronic effect of substituents on aromatic systems, a series of hypervalent iodonium salts have been synthesized (Scheme 4).

The methyl group in para position has no significant effect on the reaction and gave $\mathbf{6 b}$ in $52 \%$ yield, but the yields of $6 \mathrm{c}$ meta or $6 \mathbf{d}$ ortho methyl product were 42 and $22 \%$, respectively. However, the presence of methyl group reduced
Scheme 4. Scope of Aryl-Substituted Phenyl(vinyltrifloxy)iodonium Triflate Salts

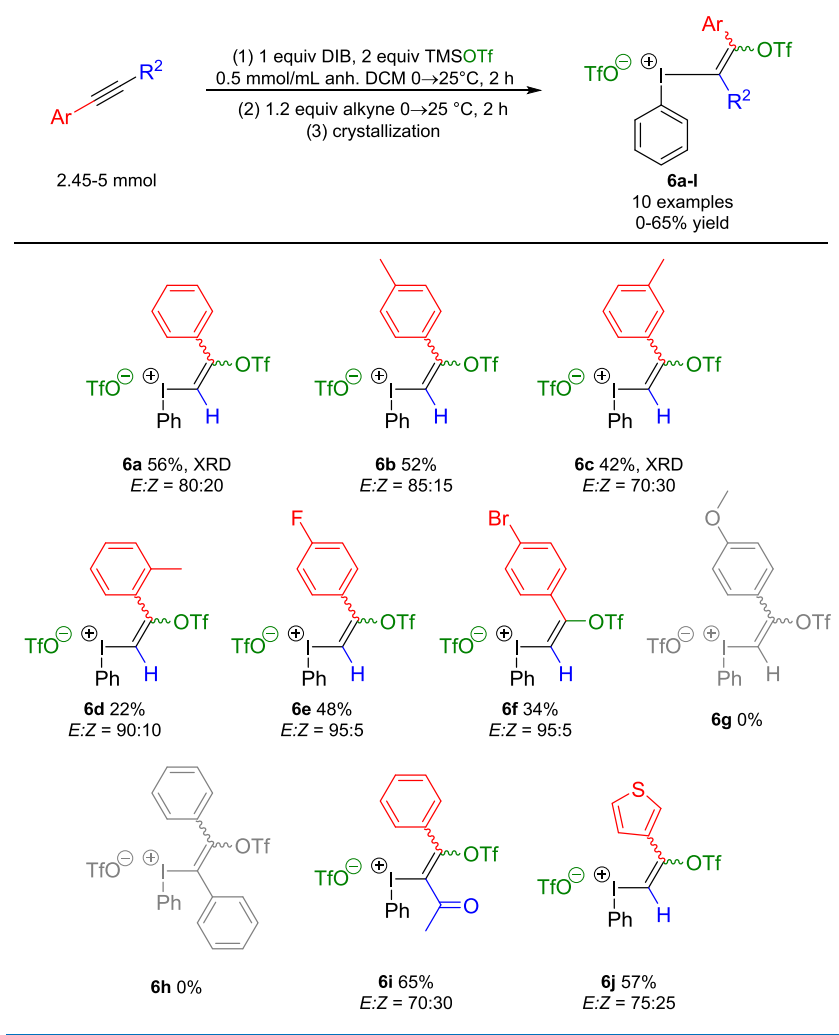

the yields, and under the reaction conditions, the formation of $E$ product gained advantage over $Z$ isomer. The structure of

Scheme 3. Study of the Conditions of the Preparation of $6 a$

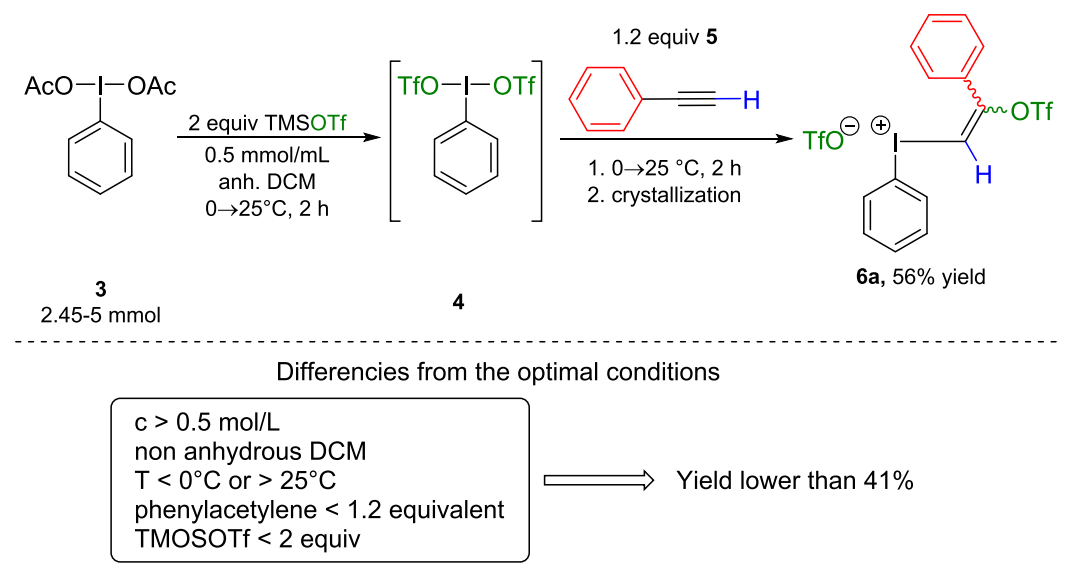


the E-6a and E-6c product has been determined also by X-ray crystallography (Figure 1). ${ }^{12}$

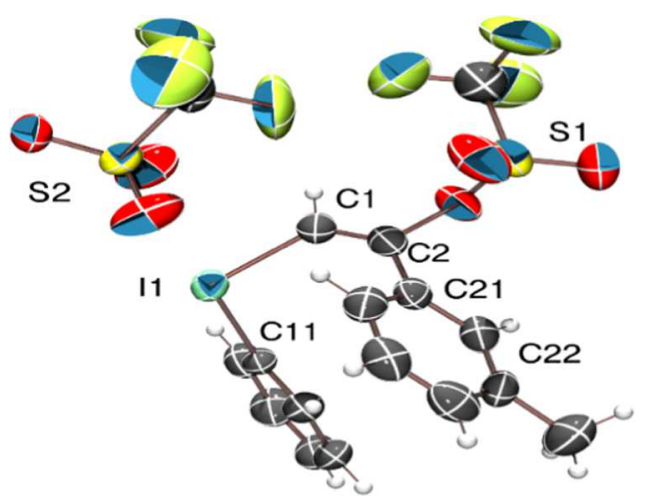

Figure 1. ORTEP view of $6 \mathrm{c}$ at $50 \%$ probability level with partial numbering scheme. Selected bond distance $[\AA]$ and bond angle [deg] data: I1-C1 2.091 (9); C1-C2 1.298 (14); I1-C11 2.112 (7); C2-C21 1.472 (13); I1-C1-C2 121.6 (7); C1-C2-C21 132.0 (9); C1-I1-C11 $97.0(3)$.

The para-fluoro $6 \mathrm{e}$ and bromo-substituted $\mathbf{6 f}$ aryl derivatives were synthesized in 48 and $34 \%$ yields, respectively. Pushing the limits further, we have experienced similar consequences in the case of the electron-rich para-methoxyphenyl $6 \mathrm{~g}$ and the bulky diphenylethyne $\mathbf{6 h}$, and no product isolated. However, the steric hindrance does not appear in the reaction of 4phenylbut-3-yn-2-one and the 6i product formed effectively with $65 \%$. There is one example to show the opportunity of the transformation of heterocyclic acetylenes: we have synthesized 3 -ethynylthiophene to the corresponding $6 \mathbf{j}$ product with $57 \%$ efficiency.

Fortunately, the reaction was not limited to the aromatic acetylenes; therefore, we investigated the synthesis of phenyl(alkenyltrifloxy)iodonium triflate salts utilizing alkyl acetylene reactants. With the best reaction conditions in hand, we started to examine the effect of structural diversity on acetylene derivatives (Scheme 5). First, we synthesized the simplest and unsubstituted phenyl(vinyltrifloxy)iodonium triflate salt (7a) by bubbling acetylene gas into the reaction mixture, which yielded the vinyl salt in $23 \%$ yield. This low yield is understandable since the product (7a) is highly soluble in most of organic solvents and decomposes quickly. Increasing the length of side chains on vinyl moiety by the application of different terminal alkylacetylenes resulted in higher stability, lower solubility, and better yields. The transformation of hex-3yne, hex-1-yne, and oct-1-yne yielded the desired products in $52 \%(7 \mathrm{~b}), 48 \%(7 \mathrm{c})$, and $66 \%(7 \mathrm{~d})$ yields, respectively. The experiments have shown that the external and internal acetylene can be utilized, and the presence of ethyl groups has no steric hindrance effect on the formation of the salts. The molecular structures of selected iodonium species were identified by X-ray measurement. $^{12}$

Next, we reacted cycloalkyl acetylenes with DIB in the presence of TMSOTf under the optimal reaction conditions. Similarly to the previous experimental experiences, we found analogous tendency of the yields as a function of ring size. The cyclopropyl-substituted phenyl(vinyl)iodonium triflate salt (7e) was obtained with only $11 \%$ yield; nevertheless, the extension of alkyl chain decreased the solubility of the salts and increased the yield as well. Accordingly, the cyclopentyl (7f,
Scheme 5. Scope of Alkyl-Substituted Phenyl(vinyltrifloxy)iodonium Triflate Salts

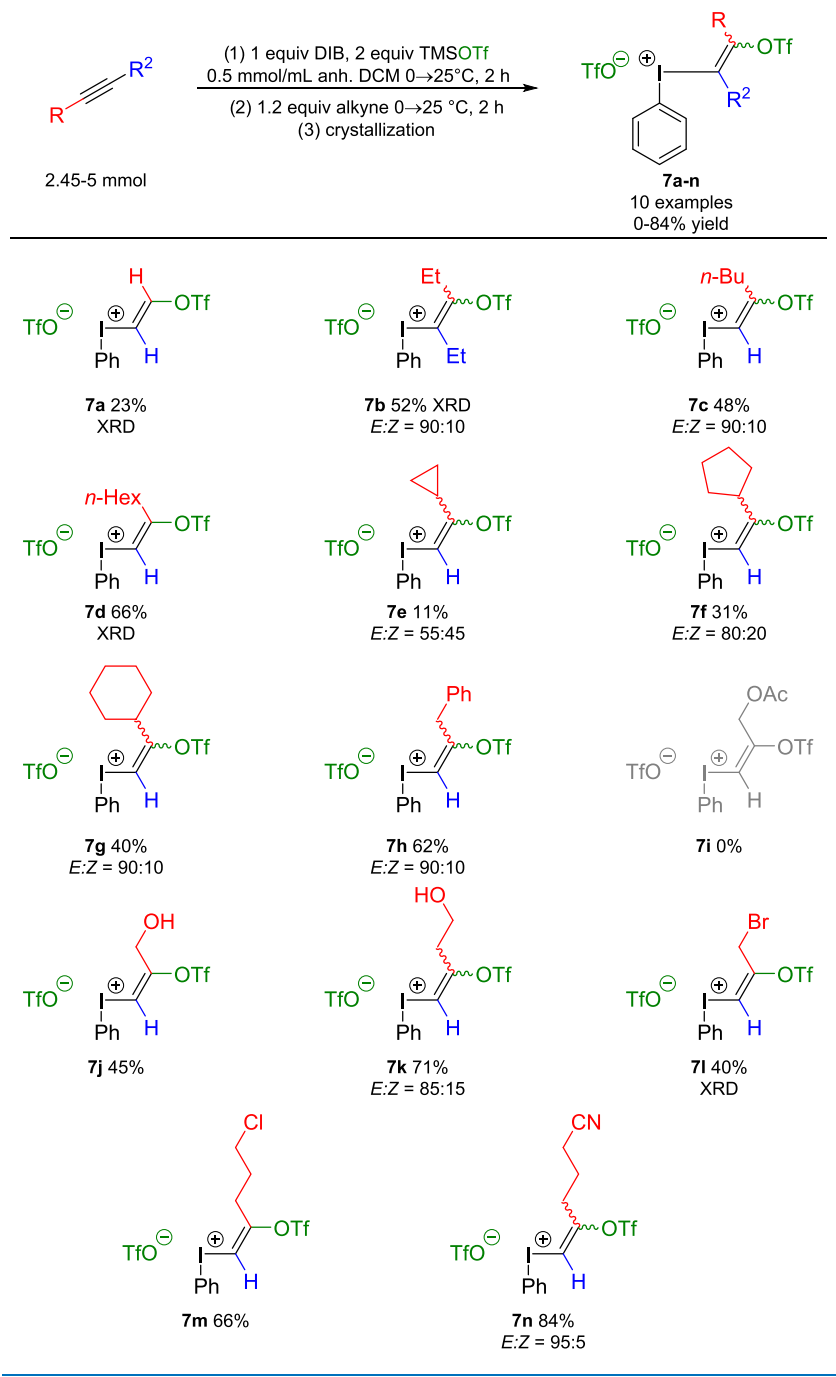

$31 \%)$, cyclohexyl (7g, 40\%), and benzyl (7h, 62\%) derivatives resulted in higher yields.

Moving forward to the synthesis of more challenging multifunctional alkenyliodonium salts, we studied the synthetic applicability of different terminal alkylacetylenes functionalized with $\mathrm{OAc}, \mathrm{OH}, \mathrm{Br}, \mathrm{Cl}$, and $\mathrm{CN}$ groups at the alkyl terminal. These functions enable further transformations on the side chain with the utilization of nucleophilic or electrophilic reagents. Although under the reaction conditions the ester functionality $(7 \mathbf{i})$ was not tolerated, the unprotected propargyl alcohol and but-3-yn-1-ol underwent reaction and gave the corresponding hypervalent iodine product $7 \mathbf{j}$ in $45 \%$ yield and $7 \mathbf{k}$ in $71 \%$ yield. Interestingly, we observed diminished stability at $25{ }^{\circ} \mathrm{C}$ in the case of $7 \mathbf{k}$ compared to $7 \mathbf{j}$, which can be rationalized by the more flexible alkyl chain and a potential intramolecular cyclization side reaction. Next, we studied the reactivity of alkynes bearing halogen and pseudohalogen functional groups. It was found that the propargyl bromide can be transformed into compound 71 with $40 \%$ yield. The structure of this trifunctional alkenyliodonium salt has been confirmed by X-ray measurements. ${ }^{12}$

The longer alkyl chain primary chloro $7 \mathrm{~m}$ and cyano $7 \mathbf{n}$ products formed in good yields, 66 and 84\%, respectively. 
As the NMR measurements revealed, under the reaction conditions, the $Z$ isomer formed in the same way as the $E$ isomer. However, the DFT calculations showed that the energy gap between the $Z$ and $E$ product is very low. ${ }^{12}$ We found that the presence of an alkyl or aryl group in (vinyltrifloxy)iodonium moiety favored in $E$ position. The separation of $Z$ and $E$ products is not feasible by crystallization.

\section{CONCLUSIONS}

In summary, we developed a new synthetic method for the preparation of substituted trifloxyvinyliodonium triflates using commercially available, easy-to-handle starting materials such as acetylene, (diacetoxyiodo)benzene, and trimethylsilyl triflate. The synthesis of the target compounds was performed under mild reaction conditions, and the procedure ensures a novel synthetic route to multifunctional alkynyliodonium salts with wide structural diversity, which were identified by multidimensional NMR measurements and X-ray crystallography. The obtained hypervalent vinyliodonium salts containing electrophilic and nucleophilic functions could serve as novel alkenyl building blocks for versatile organic transformations.

\section{EXPERIMENTAL SECTION}

General Conditions. Analytical thin-layer chromatography (TLC) was performed on Merck DC precoated TLC plates with $0.25 \mathrm{~mm}$ Kieselgel $60 \mathrm{~F} 254$. Visualization was performed with a $254 \mathrm{~nm}$ UV lamp.

The ${ }^{1} \mathrm{H},{ }^{13} \mathrm{C}$, and ${ }^{19} \mathrm{~F}$ NMR spectra were recorded on Agilent (Varian) VNMRS-400 and VNMR-600 spectrometers in $\mathrm{CD}_{2} \mathrm{Cl}_{2}$. Measurements were performed on indirect detection Z-gradient probes. Chemical shifts are expressed in parts per million $(\delta)$. The ${ }^{1} \mathrm{H}$ and ${ }^{13} \mathrm{C}$ chemical shifts are referenced to the residual solvent signals; for ${ }^{19} \mathrm{~F}$ chemical shifts, $\mathrm{CFCl}_{3}$ internal standard is used. Coupling constants $(J)$ are reported in hertz $(\mathrm{Hz})$. Splitting patterns are designated as $\mathrm{s}$ (singlet), bs (broad singlet), d (doublet), $\mathrm{t}$ (triplet), $\mathrm{q}$ (quartet), and $\mathrm{m}$ (multiplet). The structure determination is based on one- $\left({ }^{1} \mathrm{H},{ }^{13} \mathrm{C},{ }^{19} \mathrm{~F} \mathrm{NMR}\right.$, selective $1 \mathrm{~d}$-noesy) and two-dimensional $\left({ }^{1} \mathrm{H}_{-}{ }^{13} \mathrm{C}\right.$-gHSQCAD, ${ }^{1} \mathrm{H}_{-}{ }^{13} \mathrm{C}$-gHMBCAD, ${ }^{1} \mathrm{H}$-zqTOCSY) NMR experiments. NMR assignments refer in each case to the main (trans) component except in the case of $6 \mathbf{j}$ where both the trans and $Z$ isomers could be fully assigned.

IR spectra were obtained in dichloromethane solutions on a Mettler Toledo ReactIR 15, AgX DiComp probe, $6 \mathrm{~mm} \times 1.5$ $\mathrm{m}$ Fiber (silver halide), MCT detector. The in situ reactions were conducted in the following setup: sampling interval, $15 \mathrm{~s}$; $2500-650 \mathrm{~cm}^{-1}$ (resolution, $8 \mathrm{~cm}^{-1}$ ); scan option, AutoSelect; gain, $1 \times$. Data were processed by Mettler Toledo iC IR.

All melting points were measured on Büchi 501 apparatus and are uncorrected.

High-resolution mass spectra were acquired on an Agilent 6230 time-of-flight mass spectrometer equipped with a Jet Stream electrospray ion source in positive-ion mode. Injections of $0.1-0.3 \mu \mathrm{L}$ were directed to the mass spectrometer at a flow rate of $0.5 \mathrm{~mL} / \mathrm{min}$ ( $70 \%$ acetonitrile-water mixture, $0.1 \%$ formic acid), using an Agilent 1260 Infinity HPLC system. Jet Stream parameters: drying gas $\left(\mathrm{N}_{2}\right)$ flow and temperature, 10.0 $\mathrm{L} / \mathrm{min}$ and $325{ }^{\circ} \mathrm{C}$, respectively; nebulizer gas $\left(\mathrm{N}_{2}\right)$ pressure, $10 \mathrm{psi}$; capillary voltage, $4000 \mathrm{~V}$; sheath gas flow and temperature, $325^{\circ} \mathrm{C}$ and $7.5 \mathrm{~L} / \mathrm{min}$, respectively; TOFMS parameters: fragmentor voltage, $120 \mathrm{~V}$; skimmer potential, 120 V; OCT 1 RF Vpp, 750 V. Full-scan mass spectra were acquired over the $\mathrm{m} / z$ range of $100-2500$ at an acquisition rate of $250 \mathrm{~ms} / \mathrm{spectrum}$ and processed by Agilent MassHunter B.03.01 software.

Preparation of Aryl(trifloxyalkenyl)iodonium Triflate Salts. A $30 \mathrm{~mL}$ vial was charged with (diacetoxyiodo)benzene (98\%, 1 equiv, $5 \mathrm{mmol}, 1643 \mathrm{mg}$ ), equipped with a stirring bar, and then sealed with a cap. The reaction atmosphere changed to argon by three consecutive evacuation and argon backfillation processes. Then, $10 \mathrm{~mL}$ of absolute dichloromethane was added by a syringe and stirred at $25{ }^{\circ} \mathrm{C}$ for $5 \mathrm{~min}$. The solution was cooled to $0{ }^{\circ} \mathrm{C}$ and stirred vigorously for $10 \mathrm{~min}$. Trimethylsilyl trifluoromethanesulfonate (98\%, 2 equiv, 10 mmol, $2268 \mathrm{mg}, 1.85 \mathrm{~mL}$ ) was added dropwise in $1 \mathrm{~min}$. The solution turned to a clear yellow solution. The reaction mixture was allowed to warm up to $25^{\circ} \mathrm{C}$ and stirred for $2 \mathrm{~h}$. Then, the solution was cooled to $0{ }^{\circ} \mathrm{C}$ and acetylene derivative (1.2 equiv, $6 \mathrm{mmol}$ ) was added dropwise by a syringe in $2 \mathrm{~min}$ under vigorous stirring. The reaction mixture was allowed to warm up to $25{ }^{\circ} \mathrm{C}$. The yellow solution turned to a darkcolored mixture. After $2 \mathrm{~h}$, the solvent was evaporated and cold diethyl ether and pentane were added and cooled in a freezer to crystallize the salts. The precipitates were collected by filtration, and in case the solid was not white due to the decomposition products, it was washed with absolute 1,2dichloroethane (in case of aryl derivatives), diethyl ether, and pentane to obtain pure white compound. The materials were dried quickly in high vacuum at $25{ }^{\circ} \mathrm{C}$, capped tightly, and stored in a freezer at $-20{ }^{\circ} \mathrm{C}$. In these conditions, the salts remained usable for more than half of year.

6a, (E)-Phenyl(2-phenyl-2-((trifluoromethyl)sulfonyl)oxy)vinyl)iodonium trifluoromethanesulfonate ${ }^{8-10}$ and (Z)Phenyl(2-phenyl-2-(((trifluoromethyl)sulfonyl)oxy)vinyl)iodonium trifluoromethanesulfonate. The general procedure was followed starting from $5 \mathrm{mmol}$ (98\%, $1643 \mathrm{mg}$ ) (diacetoxyiodo)benzene and $6 \mathrm{mmol}(98 \%, 625 \mathrm{mg}, 672$ $\mu \mathrm{L})$ phenylacetylene; $1700 \mathrm{mg}(2.18 \mathrm{mmol}, 56 \%$ yield, $80 \% \mathrm{E}$, $10 \%$ Z) white solid. MP: $132-134{ }^{\circ} \mathrm{C}$ (dec.). ${ }^{1} \mathrm{H}$ NMR $\left(\mathrm{CD}_{2} \mathrm{Cl}_{2}, 25^{\circ} \mathrm{C}, 400 \mathrm{MHz}\right), \delta(\mathrm{ppm}): 7.39\left(2 \mathrm{H}, \mathrm{m}, \mathrm{H}^{\prime}+\right.$ $\left.\mathrm{H}^{\prime}\right)$; 7.47 (1H, s, H1); $7.48-7.56\left(4 \mathrm{H}, \mathrm{m}, \mathrm{H} 2^{\prime \prime}+\mathrm{H} 3^{\prime \prime}+\mathrm{H} 5^{\prime \prime}\right.$ $\left.+\mathrm{H}^{\prime \prime}\right) ; 7.58-7.67$ (4H, m, H4" $\left.+\mathrm{H} 2^{\prime}+\mathrm{H}^{\prime}+\mathrm{H} 6^{\prime}\right)$. ${ }^{13} \mathrm{C}\left\{{ }^{1} \mathrm{H}\right\}$ NMR $\left(\mathrm{CD}_{2} \mathrm{Cl}_{2}, 25{ }^{\circ} \mathrm{C}, 100 \mathrm{MHz}\right), \delta(\mathrm{ppm}): 95.2$ (C1); $114.8\left(\mathrm{Cl}^{\prime}\right)$; ); $118.0\left(\mathrm{q},{ }^{1} J_{\mathrm{C}, \mathrm{F}}=320.0 \mathrm{~Hz}, \mathrm{CF}_{3}\right) ; 120.5$ $\left(\mathrm{q},{ }^{1} J_{\mathrm{C}, \mathrm{F}}=320.0 \mathrm{~Hz}, \mathrm{CF}_{3}\right) ; 129.8\left(\mathrm{C} 2^{\prime \prime}+\mathrm{C}^{\prime \prime}\right) ; 130.2\left(\mathrm{C}^{\prime \prime}+\right.$ $\left.\mathrm{C5}^{\prime \prime}\right)$; $130.3\left(\mathrm{C}^{\prime \prime}\right) ; 132.6\left(\mathrm{C}^{\prime}+\mathrm{C}^{\prime}\right) ; 133.4\left(\mathrm{C}^{\prime}\right) ; 133.6$ $\left(\mathrm{C} 4^{\prime \prime}\right) ; 135.7\left(\mathrm{C} 2^{\prime}+\mathrm{C}^{\prime}\right) ; 158.6(\mathrm{C} 2) .{ }^{19} \mathrm{~F} \mathrm{NMR}\left(\mathrm{CD}_{2} \mathrm{Cl}_{2}, 25\right.$ $\left.{ }^{\circ} \mathrm{C}, 376 \mathrm{MHz}\right), \delta$ (ppm): -73.5; -79.0. IR 1430, 1266, 1240, $1221,1180,1171,1163,1139,1132,1029,988,850,779,742$, 734, 703, 677, $654 \mathrm{~cm}^{-1}$. HRMS calculated for $\mathrm{C}_{15} \mathrm{H}_{11} \mathrm{O}_{3} \mathrm{~F}_{3} \mathrm{SI}$ $[\mathrm{M}]^{+}$4549426; found 454.9422. XRD: see in SI.

6b, (E)-Phenyl(2-(p-tolyl)-2-((trifluoromethyl)sulfonyl)oxy)vinyl)iodonium Trifluoromethanesulfonate and (Z)Phenyl(2-(p-tolyl)-2-(((trifluoromethyl)sulfonyl)oxy)vinyl)iodonium Trifluoromethanesulfonate. The general procedure was followed starting from $5 \mathrm{mmol}(98 \%, 1643 \mathrm{mg})$ (diacetoxyiodo)benzene and $6 \mathrm{mmol}(98 \%, 711 \mathrm{mg}, 776$ $\mu \mathrm{L})$ 4-ethynyltoluene; $1594 \mathrm{mg}(2.58 \mathrm{mmol}, 52 \%$ yield, $85 \% \mathrm{E}$, $15 \%$ Z) white solid. MP: $121-124{ }^{\circ} \mathrm{C}$ (dec.). ${ }^{1} \mathrm{H}$ NMR $\left(\mathrm{CD}_{2} \mathrm{Cl}_{2}, 25{ }^{\circ} \mathrm{C}, 400 \mathrm{MHz}\right), \delta(\mathrm{ppm}): 2.46\left(3 \mathrm{H}, \mathrm{s}, \mathrm{CH}_{3}\right) ; 7.33$ $(1 \mathrm{H}, \mathrm{s}, \mathrm{H} 1) ; 7.34\left(2 \mathrm{H}, \mathrm{m}, \mathrm{H} 3^{\prime \prime}+\mathrm{H}^{\prime \prime}\right) ; 7.38-7.45(4 \mathrm{H}, \mathrm{m}$, $\left.\mathrm{H} 2^{\prime \prime}+\mathrm{H} 6^{\prime \prime}+\mathrm{H} 3^{\prime}+\mathrm{H} 5^{\prime}\right) ; 7.66$ (3H, m, H2 $\left.2^{\prime}+\mathrm{H}^{\prime}+\mathrm{H}^{\prime}\right)$.

${ }^{13} \mathrm{C}\left\{{ }^{1} \mathrm{H}\right\} \operatorname{NMR}\left(\mathrm{CD}_{2} \mathrm{Cl}_{2}, 25{ }^{\circ} \mathrm{C}, 100 \mathrm{MHz}\right), \delta(\mathrm{ppm}): 22.0$ 
$\left(\mathrm{CH}_{3}\right)$; $93.9(\mathrm{C} 1) ; 114.9\left(\mathrm{Cl}^{\prime}\right) ; 118.0\left(\mathrm{q},{ }^{1} J_{\mathrm{C}, \mathrm{F}}=320.0 \mathrm{~Hz}\right.$, $\left.\mathrm{CF}_{3}\right) ; 120.5\left(\mathrm{q},{ }^{1} J_{\mathrm{C}, \mathrm{F}}=320.0 \mathrm{~Hz}, \mathrm{CF}_{3}\right) ; 127.3\left(\mathrm{Cl}^{\prime \prime}\right), 129.7$ $\left(\mathrm{C} 2^{\prime \prime}+\mathrm{C}^{\prime \prime}\right) ; 130.9\left(\mathrm{C}^{\prime \prime}+\mathrm{C}^{\prime \prime}\right) ; 132.7\left(\mathrm{C}^{\prime}+\mathrm{C5}^{\prime}\right) ; 133.5$ $\left(\mathrm{C}^{\prime}\right) ; 135.7\left(\mathrm{C} 2^{\prime}+\mathrm{C}^{\prime}\right) ; 145.1\left(\mathrm{C}^{\prime \prime}\right) ; 158.9(\mathrm{C} 2) .{ }^{19} \mathrm{~F}$ NMR $\left(\mathrm{CD}_{2} \mathrm{Cl}_{2}, 25^{\circ} \mathrm{C}, 376 \mathrm{MHz}\right), \delta$ (ppm): -73.5; -79.0. IR 1424, $1255,1236,1219,1176,1133,1027,990,969,852,826,742$, 729, 716, $660 \mathrm{~cm}^{-1}$. HRMS calculated for $\mathrm{C}_{16} \mathrm{H}_{13} \mathrm{O}_{3} \mathrm{~F}_{3} \mathrm{SI}[\mathrm{M}]^{+}$ 468.9582; found 468.9576.

6c, (E)-Phenyl(2-(m-tolyl)-2-(((trifluoromethyl)sulfonyl)oxy)vinyl)iodonium Trifluoromethanesulfonate and (Z)Phenyl(2-(m-tolyl)-2-(((trifluoromethyl)sulfonyl)oxy)vinyl)iodonium Trifluoromethanesulfonate. The general procedure was followed starting from $5 \mathrm{mmol}(98 \%, 1643 \mathrm{mg}$ ) (diacetoxyiodo)benzene and $6 \mathrm{mmol}$ (97\%, $719 \mathrm{mg}, 798$ $\mu \mathrm{L})$ 3-ethynyltoluene; $1594 \mathrm{mg}(2.11 \mathrm{mmol}, 42 \%$ yield, $70 \% \mathrm{E}$, $30 \% Z$ ) white solid. MP: $127-132{ }^{\circ} \mathrm{C}$ (dec.). ${ }^{1} \mathrm{H}$ NMR $\left(\mathrm{CD}_{2} \mathrm{Cl}_{2}, 25^{\circ} \mathrm{C}, 600 \mathrm{MHz}\right), \delta(\mathrm{ppm}): 2.41\left(3 \mathrm{H}, \mathrm{s}, \mathrm{CH}_{3}\right) ; 7.24$ $\left(1 \mathrm{H}, \mathrm{s}, \mathrm{H} 2^{\prime \prime}\right)$; $7.33\left(1 \mathrm{H}, \mathrm{d}, J=7.1 \mathrm{~Hz}, \mathrm{H} 6^{\prime \prime}\right) ; 7.35$ (1H, s, H1); 7.40-7.50 (4H, m, H4" + H5 $\left.5^{\prime \prime}+\mathrm{H}^{\prime}+\mathrm{H}^{\prime}\right) ; 7.63-7.70(3 \mathrm{H}$, $\left.\mathrm{m}, \mathrm{H} 2^{\prime}+\mathrm{H} 4^{\prime}+\mathrm{H} 66^{\prime}\right) \cdot{ }^{13} \mathrm{C}\left\{{ }^{1} \mathrm{H}\right\}$ NMR $\left(\mathrm{CD}_{2} \mathrm{Cl}_{2}, 25{ }^{\circ} \mathrm{C}, 150\right.$ $\mathrm{MHz}), \delta(\mathrm{ppm}): 21.6\left(\mathrm{CH}_{3}\right) ; 94.5(\mathrm{C} 1) ; 114.8\left(\mathrm{Cl}^{\prime}\right) ; 118.0$ $\left(\mathrm{q},{ }^{1} J_{\mathrm{C}, \mathrm{F}}=320.0 \mathrm{~Hz}, \mathrm{CF}_{3}\right) ; 120.5\left(\mathrm{q},{ }^{1} J_{\mathrm{C}, \mathrm{F}}=320.0 \mathrm{~Hz}, \mathrm{CF}_{3}\right)$; $126.8\left(\mathrm{C}^{\prime \prime}\right) ; 129.9\left(\mathrm{C}^{\prime \prime}\right) ; 130.2\left(\mathrm{C}^{\prime \prime}+\mathrm{C}^{\prime \prime}\right) ; 132.8\left(\mathrm{C}^{\prime}+\right.$ $\left.\mathrm{C5}^{\prime}\right)$; $133.5\left(\mathrm{C} 4^{\prime}\right) ; 134.6\left(\mathrm{C}^{\prime \prime}\right) ; 135.7\left(\mathrm{C}^{\prime}+\mathrm{C}^{\prime}\right) ; 140.8$ (C3"), 158.7 (C2). ${ }^{19} \mathrm{~F}$ NMR $\left(\mathrm{CD}_{2} \mathrm{Cl}_{2}, 25{ }^{\circ} \mathrm{C}, 565 \mathrm{MHz}\right), \delta$ (ppm): -73.4; -79.0. IR 1430, 1268, 1243, 1223, 1180, 1169, $1161,1141,1130,1029,1010,990,895,833,742,707,680$, 673, $664 \mathrm{~cm}^{-1}$. HRMS calculated for $\mathrm{C}_{16} \mathrm{H}_{13} \mathrm{O}_{3} \mathrm{~F}_{3} \mathrm{SI}[\mathrm{M}]^{+}$ 468.9589; found 468.9582. XRD: see in the SI.

6d, (E)-Phenyl(2-(o-tolyl)-2-(((trifluoromethyl)sulfonyl)oxy)vinyl)iodonium Trifluoromethanesulfonate and (Z)Phenyl(2-(o-tolyl)-2-(((trifluoromethyl)sulfonyl)oxy)vinyl)iodonium Trifluoromethanesulfonate. The general procedure was followed starting from $3.48 \mathrm{mmol}$ (98\%, $1144 \mathrm{mg}$ ) (diacetoxyiodo)benzene and $4.18 \mathrm{mmol}(97 \%, 500 \mathrm{mg}, 542$ $\mu \mathrm{L})$ 2-ethynyltoluene; $477 \mathrm{mg}(0.77 \mathrm{mmol}, 22 \%$ yield, $90 \% \mathrm{E}$, $10 \%$ Z) white solid. MP: $98-102{ }^{\circ} \mathrm{C}$ (dec.). ${ }^{1} \mathrm{H}$ NMR $\left(\mathrm{CD}_{2} \mathrm{Cl}_{2}, 25^{\circ} \mathrm{C}, 600 \mathrm{MHz}\right), \delta(\mathrm{ppm}): 2.05\left(3 \mathrm{H}, \mathrm{s}, \mathrm{CH}_{3}\right) ; 7.29$ $\left(1 \mathrm{H}, \mathrm{d}, J=7.9 \mathrm{~Hz}, \mathrm{H} 3^{\prime \prime}\right) ; 7.36-7.40\left(2 \mathrm{H}, \mathrm{m}, \mathrm{H} 5^{\prime \prime}+\mathrm{H} 6^{\prime \prime}\right)$; $7.42\left(2 \mathrm{H}, \mathrm{m}, \mathrm{H} 3^{\prime}+\mathrm{H}^{\prime}\right) ; 7.50(1 \mathrm{H}, \mathrm{s}, \mathrm{H} 1) ; 7.50-7.60(3 \mathrm{H}$, $\left.\mathrm{m}, \mathrm{H}^{\prime \prime}+\mathrm{H} 2^{\prime}+\mathrm{H}^{\prime}\right) ; 7.67\left(1 \mathrm{H}, \mathrm{m}, \mathrm{H} 4^{\prime}\right) \cdot{ }^{13} \mathrm{C}\left\{{ }^{1} \mathrm{H}\right\} \mathrm{NMR}$ $\left(\mathrm{CD}_{2} \mathrm{Cl}_{2}, 25^{\circ} \mathrm{C}, 150 \mathrm{MHz}\right), \delta(\mathrm{ppm}): 19.4\left(\mathrm{CH}_{3}\right) ; 97.2(\mathrm{C} 1)$; $113.8\left(\mathrm{Cl}^{\prime}\right) ; 118.0\left(\mathrm{q},{ }^{1} J_{\mathrm{C}, \mathrm{F}}=320.0 \mathrm{~Hz}, \mathrm{CF}_{3}\right) ; 120.5\left(\mathrm{q},{ }^{1} J_{\mathrm{C}, \mathrm{F}}=\right.$ $\left.320.0 \mathrm{~Hz}, \mathrm{CF}_{3}\right) ; 126.9$ (C1"); 127.5 (C5"); 129.5 (C2"); 131.2 (C6"); $132.0\left(\mathrm{C}^{\prime \prime}\right)$; $132.8\left(\mathrm{C}^{\prime}+\mathrm{C}^{\prime}\right) ; 133.7\left(\mathrm{C}^{\prime \prime}\right)$; $133.8\left(\mathrm{C}^{\prime}\right) ; 136.1\left(\mathrm{C} 2^{\prime}+\mathrm{C}^{\prime}\right) ; 159.8(\mathrm{C} 2) .{ }^{19} \mathrm{~F}$ NMR $\left(\mathrm{CD}_{2} \mathrm{Cl}_{2}, 25^{\circ} \mathrm{C}, 376 \mathrm{MHz}\right), \delta$ (ppm): -73.6; -78.8. IR 1426, $1271,1240,1217,1167,1137,1109,1025,992,973,861,809$, $800,775,740,733,712,680,669,660 \mathrm{~cm}^{-1}$. HRMS calculated for $\mathrm{C}_{16} \mathrm{H}_{13} \mathrm{O}_{3} \mathrm{~F}_{3} \mathrm{SI}[\mathrm{M}]^{+} 468.9582$; found 468.9581 .

6e, (E)-(2-(4-Fluorophenyl)-2-((trifluoromethyl)sulfonyl)oxy)vinyl)(phenyl)iodonium Trifluoromethanesulfonate and (Z)-(2-(4-Fluorophenyl)-2-(((trifluoromethyl)sulfonyl)oxy)vinyl)(phenyl)iodonium Trifluoromethanesulfonate. The general procedure was followed starting from 3.33 mmol (98\%, $1094 \mathrm{mg}$ ) (diacetoxyiodo)benzene and $4 \mathrm{mmol}$ (98\%, $490 \mathrm{mg}, 467 \mu \mathrm{L}$ ) 1-ethynyl-4-fluorobenzene; $996 \mathrm{mg}$ (1.6 mmol, 48\% yield, 95\% E, 5\% Z) white solid. MP: 134$137{ }^{\circ} \mathrm{C}$ (dec.). ${ }^{1} \mathrm{H} \mathrm{NMR}\left(\mathrm{CD}_{2} \mathrm{Cl}_{2}, 25^{\circ} \mathrm{C}, 600 \mathrm{MHz}\right), \delta$ (ppm): 7.27 (2H, m, H3" + H5"); 7.39 (1H, s, H1); 7.48 (2H, m, H3' $\left.+\mathrm{H} 5^{\prime}\right) ; 7.56\left(2 \mathrm{H}, \mathrm{m}, \mathrm{H} 2^{\prime \prime}+\mathrm{H}^{\prime \prime}\right), 7.65-7.75$ (3H, m, H2' + $\left.\mathrm{H} 4^{\prime}+\mathrm{H} 66^{\prime}\right) .{ }^{13} \mathrm{C}\left\{{ }^{1} \mathrm{H}\right\}$ NMR $\left(\mathrm{CD}_{2} \mathrm{Cl}_{2}, 25{ }^{\circ} \mathrm{C}, 150 \mathrm{MHz}\right), \delta$ (ppm): $94.8(\mathrm{C} 1) ; 114.6\left(\mathrm{Cl}^{\prime}\right) ; 117.8\left(\mathrm{~d},{ }^{2} J_{\mathrm{C}, \mathrm{F}}=22.0 \mathrm{~Hz}, \mathrm{C}^{\prime \prime}\right.$
$\left.+\mathrm{C5}^{\prime \prime}\right) ; 118.0\left(\mathrm{q},{ }^{1} J_{\mathrm{C}, \mathrm{F}}=320.0 \mathrm{~Hz}, \mathrm{CF}_{3}\right) ; 120.5\left(\mathrm{q},{ }^{1} J_{\mathrm{C}, \mathrm{F}}=\right.$ $\left.320.0 \mathrm{~Hz}, \mathrm{CF}_{3}\right) ; 126.4\left(\mathrm{~d},{ }^{4} J_{\mathrm{C}, \mathrm{F}}=3.0 \mathrm{~Hz}, \mathrm{C}^{\prime \prime}\right) ; 132.4\left(\mathrm{~d},{ }^{3} \mathrm{~J}_{\mathrm{C}, \mathrm{F}}\right.$ $\left.=10.0 \mathrm{~Hz}, \mathrm{C}^{\prime \prime}+\mathrm{C}^{\prime \prime}\right) ; 133.0\left(\mathrm{C}^{\prime}+\mathrm{C}^{\prime}\right) ; 133.8\left(\mathrm{C}^{\prime}\right)$; $135.6\left(\mathrm{C} 2^{\prime}+\mathrm{C}^{\prime}\right) ; 157.7(\mathrm{C} 2) ; 165.9\left(\mathrm{~d},{ }^{1} J_{\mathrm{C}, \mathrm{F}}=254.3 \mathrm{~Hz}\right.$, $\mathrm{C} 4$ "). ${ }^{19} \mathrm{~F}$ NMR $\left(\mathrm{CD}_{2} \mathrm{Cl}_{2}, 25{ }^{\circ} \mathrm{C}, 565 \mathrm{MHz}\right), \delta$ (ppm): -73.3 ; -79.0. IR 1435, 1262, 1251, 1242, 1217, 1184, 1161, 1135, 1029, 990, 857, 844, 815, 744, 712, $656 \mathrm{~cm}^{-1}$. HRMS calculated for $\mathrm{C}_{15} \mathrm{H}_{10} \mathrm{O}_{3} \mathrm{~F}_{4} \mathrm{SI}[\mathrm{M}]^{+}$472.9332; found 472.9340 .

6f, (E)-(2-(4-Bromophenyl)-2-(((trifluoromethyl)sulfonyl)oxy)vinyl)(phenyl)iodonium Trifluoromethanesulfonate and (Z)-(2-(4-Bromophenyl)-2-(((trifluoromethyl)sulfonyl)oxy)vinyl)(phenyl)iodonium Trifluoromethanesulfonate. The general procedure was followed starting from $2.5 \mathrm{mmol}$ (98\%, $822 \mathrm{mg}$ ) (diacetoxyiodo)benzene and $3 \mathrm{mmol}(98 \%$, $554 \mathrm{mg}$ ) 1-bromo-4-ethynylbenzene; $576 \mathrm{mg}$ (0.84 mmol, 34\% yield, 95\% E, 5\% Z) white solid. MP: $143-147{ }^{\circ} \mathrm{C}$ (dec.). ${ }^{1} \mathrm{H}$ $\operatorname{NMR}\left(\mathrm{CD}_{2} \mathrm{Cl}_{2}, 25^{\circ} \mathrm{C}, 400 \mathrm{MHz}\right), \delta(\mathrm{ppm}): 7.38\left(2 \mathrm{H}, \mathrm{m}, \mathrm{H} 2^{\prime \prime}\right.$ + H6 $\left.{ }^{\prime \prime}\right) ; 7.43$ (2H, m, H3 $\left.{ }^{\prime}+\mathrm{H}^{\prime}\right)$; 7.46 (1H, s, H1); 7.60$7.72\left(5 \mathrm{H}, \mathrm{m}, \mathrm{H} 2^{\prime}+\mathrm{H} 4^{\prime}+\mathrm{H} 6^{\prime}+\mathrm{H} 3^{\prime \prime}+\mathrm{H}^{\prime \prime}\right) .{ }^{13} \mathrm{C}\left\{{ }^{1} \mathrm{H}\right\} \mathrm{NMR}$ $\left(\mathrm{CD}_{2} \mathrm{Cl}_{2}, 25^{\circ} \mathrm{C}, 100 \mathrm{MHz}\right), \delta(\mathrm{ppm}): 95.6(\mathrm{C} 1) ; 114.8\left(\mathrm{Cl}^{\prime}\right)$; $118.0\left(\mathrm{q},{ }^{1} J_{\mathrm{C}, \mathrm{F}}=320.0 \mathrm{~Hz}, \mathrm{CF}_{3}\right) ; 120.5\left(\mathrm{q},{ }^{1} J_{\mathrm{C}, \mathrm{F}}=320.0 \mathrm{~Hz}\right.$, $\left.\mathrm{CF}_{3}\right) ; 128.6+129.2\left(\mathrm{C} 1^{\prime \prime}+\mathrm{C} 4^{\prime \prime}\right) ; 131.3\left(\mathrm{C}^{\prime \prime}+\mathrm{C}^{\prime \prime}\right) ; 132.8$ $\left(\mathrm{C}^{\prime}+\mathrm{C}^{\prime}\right) ; 133.6\left(\mathrm{C}^{\prime}+\mathrm{C}^{\prime \prime}+\mathrm{C}^{\prime \prime}\right) ; 135.6\left(\mathrm{C}^{\prime}+\mathrm{C}^{\prime}\right)$, 157.5 (C2). ${ }^{19} \mathrm{~F}$ NMR $\left(\mathrm{CD}_{2} \mathrm{Cl}_{2}, 25{ }^{\circ} \mathrm{C}, 376 \mathrm{MHz}\right), \delta(\mathrm{ppm})$ : -73.3; -79.0. IR 1426, 1264, 1242, 1234, 1227, 1174, 1133, 1027, 1014, 992, 979, 850, 738, 693, $654 \mathrm{~cm}^{-1}$. HRMS calculated for $\mathrm{C}_{15} \mathrm{H}_{10} \mathrm{O}_{3} \mathrm{~F}_{3} \mathrm{SBrI}[\mathrm{M}]^{+}$532.8531; found 532.8531.

6i, (E)-(3-Oxo-1-phenyl-1-(((trifluoromethyl)sulfonyl)oxy)but-1-en-2-yl)(phenyl)iodonium Trifluoromethanesulfonate and (Z)-(3-Oxo-1-phenyl-1-(((trifluoromethyl)sulfonyl)oxy)but-1-en-2-yl)(phenyl)iodonium Trifluoromethanesulfonate. The general procedure was followed starting from $5 \mathrm{mmol}$ $(98 \%, 1643 \mathrm{mg}$ ) (diacetoxyiodo)benzene and $6 \mathrm{mmol}(96 \%$, $901 \mathrm{mg}, 910 \mu \mathrm{L})$ 4-phenyl-3-butyn-2-one; $2111 \mathrm{mg}$ (3.27 mmol, $65 \%$ yield, $70 \%$ E, 30\% Z) white solid. MP: $125-128$ ${ }^{\circ} \mathrm{C}$ (dec.). ${ }^{1} \mathrm{H}$ NMR $\left(\mathrm{CD}_{2} \mathrm{Cl}_{2}, 25{ }^{\circ} \mathrm{C}, 400 \mathrm{MHz}\right), \delta$ (ppm): $2.77\left(3 \mathrm{H}, \mathrm{s}, \mathrm{CH}_{3}\right) ; 7.40\left(2 \mathrm{H}, \mathrm{m}, \mathrm{H}^{\prime}+\mathrm{H}^{\prime}\right) ; 7.47(2 \mathrm{H}, \mathrm{m}$, $\left.\mathrm{H} 2^{\prime \prime}+\mathrm{H}^{\prime \prime}\right), 7.50-7.60\left(4 \mathrm{H}, \mathrm{m}, \mathrm{H} 2^{\prime}+\mathrm{H}^{\prime}+\mathrm{H}^{\prime \prime}+\mathrm{H} 5^{\prime \prime}\right)$; $7.63\left(1 \mathrm{H}, \mathrm{m}, \mathrm{H} 4^{\prime}\right) ; 7.70\left(1 \mathrm{H}, \mathrm{m}, \mathrm{H} 4^{\prime \prime}\right) .{ }^{13} \mathrm{C}\left\{{ }^{1} \mathrm{H}\right\}$ NMR $\left(\mathrm{CD}_{2} \mathrm{Cl}_{2}, 25{ }^{\circ} \mathrm{C}, 100 \mathrm{MHz}\right), \delta(\mathrm{ppm}): 30.0(\mathrm{C} 4) ; 114.8\left(\mathrm{Cl}^{\prime}\right)$; $118.0\left(\mathrm{q},{ }^{1} J_{\mathrm{C}, \mathrm{F}}=320.0 \mathrm{~Hz}, \mathrm{CF}_{3}\right) ; 120.5\left(\mathrm{q},{ }^{1} J_{\mathrm{C}, \mathrm{F}}=320.0 \mathrm{~Hz}\right.$, $\left.\mathrm{CF}_{3}\right) ; 125.8(\mathrm{C} 2) ; 128.4\left(\mathrm{C} 1^{\prime \prime}\right) ; 129.9\left(\mathrm{C}^{\prime \prime}+\mathrm{C}^{\prime \prime}\right) ; 130.2$ $\left(\mathrm{C} 2^{\prime \prime}+\mathrm{C}^{\prime \prime}\right) ; 132.4\left(\mathrm{C}^{\prime}+\mathrm{C}^{\prime}\right) ; 133.5\left(\mathrm{C}^{\prime}\right) ; 134.1\left(\mathrm{C} 4^{\prime \prime}\right)$; $136.5\left(\mathrm{C} 2^{\prime}+\mathrm{C}^{\prime}\right) ; 159.9$ (C1); 191.3 (C3). ${ }^{19} \mathrm{~F}$ NMR $\left(\mathrm{CD}_{2} \mathrm{Cl}_{2}, 25{ }^{\circ} \mathrm{C}, 376 \mathrm{MHz}\right), \delta(\mathrm{ppm}):-72.6 ;-78.8$. IR 1445, $1434,1279,1219,1169,1130,1038,1022,984,934,913,880$, $844,783,766,736,703,686,677 \mathrm{~cm}^{-1}$. HRMS calculated for $\mathrm{C}_{17} \mathrm{H}_{13} \mathrm{O}_{4} \mathrm{~F}_{3} \mathrm{SI}[\mathrm{M}]^{+}$496.9526; found 496.9531 .

6j, (E)-Phenyl(2-(thiophen-3-yl)-2-((trifluoromethyl)sulfonyl)oxy)vinyl)iodonium Trifluoromethanesulfonate and (Z)-Phenyl(2-(thiophen-3-yl)-2-(((trifluoromethyl)sulfonyl)oxy)vinyl)iodonium Trifluoromethanesulfonate. The general procedure was followed starting from 3.85 $\mathrm{mmol}(98 \%, 1265 \mathrm{mg}$ ) (diacetoxyiodo)benzene and 4.62 mmol (97\%, $515 \mathrm{mg}, 474 \mu \mathrm{L}$ ) 3-ethynyltiophene; $1347 \mathrm{mg}$ (2.21 mmol, $57 \%$ yield, $75 \% \mathrm{E}, 25 \% \mathrm{Z}$ ) brownish deep green solid, MP: $84-87{ }^{\circ} \mathrm{C}$ (dec.). E-isomer: ${ }^{1} \mathrm{H}$ NMR $\left(\mathrm{CD}_{2} \mathrm{Cl}_{2}, 25\right.$ $\left.{ }^{\circ} \mathrm{C}, 400 \mathrm{MHz}\right), \delta(\mathrm{ppm}): 7.30\left(1 \mathrm{H}, \mathrm{dd}, J=5.1,1.3 \mathrm{~Hz}, \mathrm{H} 4{ }^{\prime \prime}\right)$; 7.35 (1H, s, H1); $7.44\left(2 \mathrm{H}, \mathrm{m}, \mathrm{H} 3^{\prime}+\mathrm{H}^{\prime}\right) ; 7.56(1 \mathrm{H}, \mathrm{dd}, J=$ 5.1, $\left.3.0 \mathrm{~Hz}, \mathrm{H} 5^{\prime \prime}\right) ; 7.64\left(1 \mathrm{H}, \mathrm{m}, \mathrm{H} 4^{\prime}\right) ; 7.72\left(2 \mathrm{H}, \mathrm{m}, \mathrm{H} 2^{\prime}+\right.$ $\left.\mathrm{H}^{\prime}\right)$; $7.96\left(1 \mathrm{H}, \mathrm{dd}, J=3.0,1.3 \mathrm{~Hz}, \mathrm{H} 2^{\prime \prime}\right) .{ }^{13} \mathrm{C}\left\{{ }^{1} \mathrm{H}\right\} \mathrm{NMR}$ $\left(\mathrm{CD}_{2} \mathrm{Cl}_{2}, 25^{\circ} \mathrm{C}, 100 \mathrm{MHz}\right), \delta(\mathrm{ppm}): 93.2(\mathrm{C} 1) ; 114.6\left(\mathrm{Cl}^{\prime}\right)$; 
$118.0\left(\mathrm{q},{ }^{1} J_{\mathrm{C}, \mathrm{F}}=320.0 \mathrm{~Hz}, \mathrm{CF}_{3}\right) ; 120.5\left(\mathrm{q},{ }^{1} J_{\mathrm{C}, \mathrm{F}}=320.0 \mathrm{~Hz}\right.$, $\left.\mathrm{CF}_{3}\right) ; 127.4\left(\mathrm{C}^{\prime \prime}\right) ; 129.6\left(\mathrm{C}^{\prime \prime}\right) ; 132.9\left(\mathrm{C}^{\prime}+\mathrm{C}^{\prime}\right) ; 133.1$ $\left(\mathrm{C} 2^{\prime \prime}\right) ; 133.6\left(\mathrm{C}^{\prime}\right) ; 135.5\left(\mathrm{C}^{\prime}+\mathrm{C}^{\prime}\right) ; 138.0\left(\mathrm{C}^{\prime \prime}\right) ; 154.0$ (C2). ${ }^{19} \mathrm{~F}$ NMR $\left(\mathrm{CD}_{2} \mathrm{Cl}_{2}, 25{ }^{\circ} \mathrm{C}, 376 \mathrm{MHz}\right), \delta(\mathrm{ppm}):-72.5$; -78.9. Z-isomer: ${ }^{1} \mathrm{H}$ NMR $\left(\mathrm{CD}_{2} \mathrm{Cl}_{2}, 25{ }^{\circ} \mathrm{C}, 400 \mathrm{MHz}\right), \delta$ (ppm): 7.30 (1H, dd, $\left.J=5.1,1.2 \mathrm{~Hz}, \mathrm{H} 4^{\prime \prime}\right) ; 7.47(1 \mathrm{H}, \mathrm{s}, \mathrm{H1})$; 7.48 (1H, dd, $\left.J=5.1,2.8 \mathrm{~Hz}, \mathrm{H}^{\prime \prime}\right)$; $7.54\left(2 \mathrm{H}, \mathrm{m}, \mathrm{H}^{\prime}+\mathrm{H}^{\prime}\right)$; $7.70\left(1 \mathrm{H}, \mathrm{m}, \mathrm{H} 4^{\prime}\right) ; 7.85\left(1 \mathrm{H}, \mathrm{dd}, J=2.8,1.2 \mathrm{~Hz}, \mathrm{H} 2^{\prime \prime}\right) ; 8.08$ $\left(2 \mathrm{H}, \mathrm{m}, \mathrm{H} 2^{\prime}+\mathrm{H}^{\prime}\right) \cdot{ }^{13} \mathrm{C}\left\{{ }^{1} \mathrm{H}\right\}$ NMR $\left(\mathrm{CD}_{2} \mathrm{Cl}_{2}, 25{ }^{\circ} \mathrm{C}, 100\right.$ $\mathrm{MHz}), \delta(\mathrm{ppm}): 89.2(\mathrm{C} 1) ; 114.1\left(\mathrm{Cl}^{\prime}\right) ; 118.0\left(\mathrm{q},{ }^{1} J_{\mathrm{C}, \mathrm{F}}=\right.$ $\left.320.0 \mathrm{~Hz}, \mathrm{CF}_{3}\right) ; 120.5$ (q, $\left.{ }^{1} J_{\mathrm{C}, \mathrm{F}}=320.0 \mathrm{~Hz}, \mathrm{CF}_{3}\right) ; 126.3\left(\mathrm{C4}^{\prime \prime}\right)$; $129.1\left(\mathrm{C5}^{\prime \prime}\right) ; 131.1\left(\mathrm{C} 2^{\prime \prime}\right)$; $133.1\left(\mathrm{C}^{\prime}+\mathrm{C}^{\prime}\right) ; 133.7\left(\mathrm{C}^{\prime}\right)$; $136.0\left(\mathrm{C} 2^{\prime}+\mathrm{C}^{\prime}\right) ; 138.0\left(\mathrm{C}^{\prime \prime}\right) ; 153.8$ (C2). ${ }^{19} \mathrm{~F}$ NMR $\left(\mathrm{CD}_{2} \mathrm{Cl}_{2}, 25{ }^{\circ} \mathrm{C}, 376 \mathrm{MHz}\right), \delta$ (ppm): -73.5; -78.9. E-Z: IR $1430,1275,1238,1217,1167,1152,1137,1025,1003,990$, 913, 878, 848, 822, 807, 787, 762, 748, 733, 708, $677 \mathrm{~cm}^{-1}$. HRMS calculated for $\mathrm{C}_{13} \mathrm{H}_{9} \mathrm{O}_{3} \mathrm{~F}_{3} \mathrm{~S}_{2} \mathrm{I}[\mathrm{M}]^{+}$460.8988; found 460.8990.

7a, (E)-Phenyl(2-(((trifluoromethyl)sulfonyl)oxy)vinyl)iodonium Trifluoromethanesulfonate. ${ }^{6 a, 7 b, 8,10}$ The general procedure was followed starting from $5 \mathrm{mmol}(98 \%, 1643 \mathrm{mg}$ ) (diacetoxyiodo)benzene. Acetylene gas was generated from 6 mmol $\mathrm{CaC}_{2}$ (80\%, $\left.481 \mathrm{mg}\right) ; 545 \mathrm{mg}$ (1.03 mmol, $21 \%$ yield) white solid. MP: $110-116{ }^{\circ} \mathrm{C}$ (dec.). ${ }^{1} \mathrm{H}$ NMR $\left(\mathrm{CD}_{2} \mathrm{Cl}_{2}, 25\right.$ $\left.{ }^{\circ} \mathrm{C}, 400 \mathrm{MHz}\right), \delta(\mathrm{ppm}): 7.09(1 \mathrm{H}, \mathrm{d}, J=12.3 \mathrm{~Hz}, \mathrm{H1}) ; 7.54$ $\left(2 \mathrm{H}, \mathrm{m}, \mathrm{H} 3^{\prime}+\mathrm{H} 5^{\prime}\right), 7.68(1 \mathrm{H}, \mathrm{d}, J=12.3 \mathrm{~Hz}, \mathrm{H} 2) ; 7.72(1 \mathrm{H}$, m, H4 $\left.4^{\prime}\right) ; 8.03\left(2 \mathrm{H}, \mathrm{m}, \mathrm{H} 2^{\prime}+\mathrm{H}^{\prime}\right) \cdot{ }^{13} \mathrm{C}\left\{{ }^{1} \mathrm{H}\right\} \mathrm{NMR}\left(\mathrm{CD}_{2} \mathrm{Cl}_{2}\right.$, $\left.25{ }^{\circ} \mathrm{C}, 100 \mathrm{MHz}\right), \delta(\mathrm{ppm}): 91.3(\mathrm{C} 1) ; 113.1\left(\mathrm{Cl}^{\prime}\right) ; 118.0(\mathrm{q}$, $\left.{ }^{1} J_{\mathrm{C}, \mathrm{F}}=320.0 \mathrm{~Hz}, \mathrm{CF}_{3}\right) ; 120.5\left(\mathrm{q},{ }^{1} J_{\mathrm{C}, \mathrm{F}}=320.0 \mathrm{~Hz}, \mathrm{CF}_{3}\right) ; 133.0$ $\left(\mathrm{C}^{\prime}+\mathrm{C}^{\prime}\right) ; 133.7\left(\mathrm{C}^{\prime}\right) ; 136.1\left(\mathrm{C}^{\prime}+\mathrm{C}^{\prime}\right) ; 149.9(\mathrm{C} 2) .{ }^{19} \mathrm{~F}$ NMR $\left(\mathrm{CD}_{2} \mathrm{Cl}_{2}, 25^{\circ} \mathrm{C}, 376 \mathrm{MHz}\right), \delta(\mathrm{ppm}):-73.3$; -79.0. IR $1422,1249,1219,1180,1169,1133,1022,1008,990,906$, $856,761,740,686,679, \mathrm{~cm}^{-1}$. HRMS calculated for $\mathrm{C}_{9} \mathrm{H}_{7} \mathrm{O}_{3} \mathrm{~F}_{3} \mathrm{SI}[\mathrm{M}]^{+}$378.9113; found 378.9107. XRD: see in the SI.

7b, (E)-Phenyl(4-((trifluoromethyl)sulfonyl)oxy)hex-3-en3-yl)iodonium Trifluoromethanesulfonate ${ }^{10}$ and (Z)-Phenyl(4-(((trifluoromethyl)sulfonyl)oxy)hex-3-en-3-yl)iodonium Trifluoromethanesulfonate. The general procedure was followed starting from $5 \mathrm{mmol}(98 \%, 1643 \mathrm{mg})$ (diacetoxyiodo)benzene and $6 \mathrm{mmol}$ (97\%, $508 \mathrm{mg}, 703$ $\mu \mathrm{L})$ 3-hexyne; $1531 \mathrm{mg}(2.62 \mathrm{mmol}, 52 \%$ yield, $90 \% \mathrm{E}, 10 \%$ $Z)$ white solid. MP: $85-87{ }^{\circ} \mathrm{C}$ (dec.). ${ }^{1} \mathrm{H}$ NMR $\left(\mathrm{CD}_{2} \mathrm{Cl}_{2}, 25\right.$ $\left.{ }^{\circ} \mathrm{C}, 400 \mathrm{MHz}\right), \delta(\mathrm{ppm}): 1.19\left(3 \mathrm{H}, \mathrm{t}, J=7.1 \mathrm{~Hz}, \mathrm{CH}_{3}\right) ; 1.33$ $\left(3 \mathrm{H}, \mathrm{t}, J=7.1 \mathrm{~Hz}, \mathrm{CH}_{3}\right) ; 2.76\left(2 \mathrm{H}, \mathrm{q}, J=7.1 \mathrm{~Hz}, \mathrm{CH}_{2}\right) ; 3.04$ $\left(2 \mathrm{H}, \mathrm{q}, J=7.1 \mathrm{~Hz}, \mathrm{CH}_{2}\right) ; 7.55\left(2 \mathrm{H}, \mathrm{m}, \mathrm{H} 3^{\prime}+\mathrm{H}^{\prime}\right), 7.71(1 \mathrm{H}$, m, H4 $\left.4^{\prime}\right) ; 7.95\left(2 \mathrm{H}, \mathrm{m}, \mathrm{H} 2^{\prime}+\mathrm{H}^{\prime}\right) \cdot{ }^{13} \mathrm{C}\left\{{ }^{1} \mathrm{H}\right\} \mathrm{NMR}\left(\mathrm{CD}_{2} \mathrm{Cl}_{2}\right.$, $\left.25^{\circ} \mathrm{C}, 100 \mathrm{MHz}\right), \delta(\mathrm{ppm}): 11.3\left(\mathrm{CH}_{3}\right) ; 13.7\left(\mathrm{CH}_{3}\right) ; 28.7$ $\left(\mathrm{CH}_{2}\right) ; 30.8\left(\mathrm{CH}_{2}\right) ; 112.6\left(\mathrm{Cl}^{\prime}\right) ; 118.0\left(\mathrm{q},{ }^{1} J_{\mathrm{C}, \mathrm{F}}=320.0 \mathrm{~Hz}\right.$, $\left.\mathrm{CF}_{3}\right) ; 120.5\left(\mathrm{q},{ }^{1} J_{\mathrm{C}, \mathrm{F}}=320.0 \mathrm{~Hz}, \mathrm{CF}_{3}\right) ; 123.8(\mathrm{C} 3) ; 133.0$ $\left(\mathrm{C}^{\prime}+\mathrm{C}^{\prime}\right) ; 133.5\left(\mathrm{C}^{\prime}\right) ; 135.4\left(\mathrm{C}^{\prime}+\mathrm{C}^{\prime}\right) ; 157.4(\mathrm{C} 4) .{ }^{19} \mathrm{~F}$ NMR $\left(\mathrm{CD}_{2} \mathrm{Cl}_{2}, 25^{\circ} \mathrm{C}, 376 \mathrm{MHz}\right), \delta$ (ppm): -72.3; -76.9. IR $1415,1279,1234,1223,1206,1165,1146,1132,1085,1025$, $1010,990,956,878,742,680,665 \mathrm{~cm}^{-1}$. HRMS calculated for $\mathrm{C}_{13} \mathrm{H}_{15} \mathrm{O}_{3} \mathrm{~F}_{3} \mathrm{SI}[\mathrm{M}]^{+}$434.9739; found 434.9746. XRD: see in the SI.

7c, (E)-Phenyl(2-(((trifluoromethyl)sulfonyl)oxy)hex-1-en1-yl)iodonium Trifluoromethanesulfonate ${ }^{6 a, e, f, 8,10}$ and (Z)Phenyl(2-(((trifluoromethyl)sulfonyl)oxy)hex-1-en-1-yl)iodonium Trifluoromethanesulfonate. The general procedure was followed starting from $5 \mathrm{mmol}(98 \%, 1643 \mathrm{mg}$ ) (diacetoxyiodo)benzene and $6 \mathrm{mmol}$ (97\%, $508 \mathrm{mg}, 711$ $\mu \mathrm{L})$ 1-hexyne; $1390 \mathrm{mg}$ ( $2.38 \mathrm{mmol}, 48 \%$ yield, $90 \% \mathrm{E}, 10 \%$
$Z)$ white solid. MP: $114-118{ }^{\circ} \mathrm{C}$ (dec.). ${ }^{1} \mathrm{H}$ NMR $\left(\mathrm{CD}_{2} \mathrm{Cl}_{2}\right.$, $\left.25{ }^{\circ} \mathrm{C}, 400 \mathrm{MHz}\right), \delta(\mathrm{ppm}): 0.91\left(3 \mathrm{H}, \mathrm{m}, \mathrm{CH}_{3}\right) ; 1.37(2 \mathrm{H}, \mathrm{m}$, $\left.\mathrm{H} 5_{\mathrm{x}, \mathrm{y}}\right) ; 2.18\left(2 \mathrm{H}, \mathrm{m}, \mathrm{H} 4_{\mathrm{x}, \mathrm{y}}\right)$; $2.82\left(2 \mathrm{H}, \mathrm{t}, J=7.2 \mathrm{~Hz}, \mathrm{H} 3_{\mathrm{x}, \mathrm{y}}\right)$; $7.06(1 \mathrm{H}$, br. s, $\mathrm{H} 1)$; $7.53\left(2 \mathrm{H}, \mathrm{m}, \mathrm{H} 3^{\prime}+\mathrm{H} 5^{\prime}\right), 7.71(1 \mathrm{H}, \mathrm{m}$, $\left.\mathrm{H} 4^{\prime}\right) ; 7.98\left(2 \mathrm{H}, \mathrm{m}, \mathrm{H} 2^{\prime}+\mathrm{H} 6^{\prime}\right) .{ }^{13} \mathrm{C}\left\{{ }^{1} \mathrm{H}\right\} \mathrm{NMR}\left(\mathrm{CD}_{2} \mathrm{Cl}_{2}, 25\right.$ $\left.{ }^{\circ} \mathrm{C}, 100 \mathrm{MHz}\right), \delta(\mathrm{ppm}): 13.9\left(\mathrm{CH}_{3}\right) ; 22.6(\mathrm{C} 5) ; 28.6(\mathrm{C} 4)$; 35.2 (C3); 92.1 (C1); $114.3\left(\mathrm{Cl}^{\prime}\right) ; 118.0\left(\mathrm{q},{ }^{1} J_{\mathrm{C}, \mathrm{F}}=320.0 \mathrm{~Hz}\right.$, $\left.\mathrm{CF}_{3}\right) ; 120.5\left(\mathrm{q},{ }^{1} J_{\mathrm{C}, \mathrm{F}}=320.0 \mathrm{~Hz}, \mathrm{CF}_{3}\right) ; 133.2\left(\mathrm{C}^{\prime}+\mathrm{C}^{\prime}\right)$; $133.7\left(\mathrm{C}^{\prime}\right) ; 135.3\left(\mathrm{C} 2^{\prime}+\mathrm{C}^{\prime}\right) ; 163.4(\mathrm{C} 2) .{ }^{19} \mathrm{~F}$ NMR $\left(\mathrm{CD}_{2} \mathrm{Cl}_{2}, 25{ }^{\circ} \mathrm{C}, 376 \mathrm{MHz}\right), \delta(\mathrm{ppm}):-73.6 ;-78.9$. IR 1430, $1269,1242,1223,1210,1165,1139,1122,1061,1023,984$, $900,856,828,755,742,731,675,654 \mathrm{~cm}^{-1}$. HRMS calculated for $\mathrm{C}_{13} \mathrm{H}_{15} \mathrm{O}_{3} \mathrm{~F}_{3} \mathrm{SI}[\mathrm{M}]^{+}$434.9739; found 434.9742.

7d, (E)-Phenyl(2-(((trifluoromethyl)sulfonyl)oxy)oct-1-en1-yl)iodonium Trifluoromethanesulfonate. ${ }^{6 a, 9,10}$ The general procedure was followed starting from $5 \mathrm{mmol}(98 \%, 1643 \mathrm{mg}$ ) (diacetoxyiodo)benzene and $6 \mathrm{mmol}(99 \%, 668 \mathrm{mg}, 894 \mu \mathrm{L}$ ) 1-octyne; $2030 \mathrm{mg}$ (3.32 mmol, 66\% yield) white solid. MP: 121-123 ${ }^{\circ} \mathrm{C}$ (dec.). ${ }^{1} \mathrm{H}$ NMR $\left(\mathrm{CD}_{2} \mathrm{Cl}_{2}, 25{ }^{\circ} \mathrm{C}, 400 \mathrm{MHz}\right), \delta$ (ppm): $0.88\left(3 \mathrm{H}, \mathrm{t}, J=6.9 \mathrm{~Hz}, \mathrm{CH}_{3}\right) ; 1.20-1.40\left(6 \mathrm{H}, \mathrm{m}, \mathrm{H} 7_{\mathrm{x}, \mathrm{y}}\right.$ $\left.+\mathrm{H}_{\mathrm{x}, \mathrm{y}}+\mathrm{H} 5_{\mathrm{x}, \mathrm{y}}\right) ; 1.53\left(2 \mathrm{H}, \mathrm{m}, \mathrm{H} 4_{\mathrm{x}, \mathrm{y}}\right) ; 2.81(2 \mathrm{H}, \mathrm{t}, J=7.6 \mathrm{~Hz}$, $\left.\mathrm{H} 3_{\mathrm{x}, \mathrm{y}}\right) ; 7.14$ (1H, s, H1); $7.53\left(2 \mathrm{H}, \mathrm{m}, \mathrm{H} 3^{\prime}+\mathrm{H}^{\prime}\right) ; 7.69(1 \mathrm{H}$, m, H4 $\left.4^{\prime}\right) ; 8.00\left(2 \mathrm{H}, \mathrm{m}, \mathrm{H} 2^{\prime}+\mathrm{H}^{\prime}\right) \cdot{ }^{13} \mathrm{C}\left\{{ }^{1} \mathrm{H}\right\} \mathrm{NMR}\left(\mathrm{CD}_{2} \mathrm{Cl}_{2}\right.$, $\left.25{ }^{\circ} \mathrm{C}, 100 \mathrm{MHz}\right), \delta(\mathrm{ppm}): 14.2\left(\mathrm{CH}_{3}\right) ; 22.9+29.0+31.8$ $(\mathrm{C} 7$ + C6 + C5); $26.6(\mathrm{C} 4) ; 35.4$ (C3); 93.0 (C1); 114.5 $\left(\mathrm{C}^{\prime}\right) ; 118.9\left(\mathrm{q},{ }^{1} J_{\mathrm{C}, \mathrm{F}}=320.0 \mathrm{~Hz}, \mathrm{CF}_{3}\right) ; 120.6\left(\mathrm{q},{ }^{1} J_{\mathrm{C}, \mathrm{F}}=320.0\right.$ $\left.\mathrm{Hz}, \mathrm{CF}_{3}\right) ; 133.0\left(\mathrm{C}^{\prime}+\mathrm{C5}^{\prime}\right) ; 133.4\left(\mathrm{C}^{\prime}\right) ; 135.3\left(\mathrm{C}^{\prime}+\mathrm{C}^{\prime}\right)$; $163.3(\mathrm{C} 2) .{ }^{19} \mathrm{~F}$ NMR $\left(\mathrm{CD}_{2} \mathrm{Cl}_{2}, 25{ }^{\circ} \mathrm{C}, 376 \mathrm{MHz}\right), \delta(\mathrm{ppm})$ : -71.7; -77.0. IR 1432, 1271, 1245, 1221, 1208, 1163, 1143, $1128,1027,951,899,856,835,751,731,675,654 \mathrm{~cm}^{-1}$. HRMS calculated for $\mathrm{C}_{15} \mathrm{H}_{19} \mathrm{O}_{3} \mathrm{~F}_{3} \mathrm{SI}[\mathrm{M}]^{+} 434.9739$; found 434.9742. XRD: see in the SI.

7e, (E)-(2-Cyclopropyl-2-(((trifluoromethyl)sulfonyl)oxy)vinyl)(phenyl)iodonium Trifluoromethanesulfonate and (Z)-(2-Cyclopropyl-2-(((trifluoromethyl)sulfonyl)oxy)vinyl)(phenyl)iodonium Trifluoromethanesulfonate. The general procedure was followed starting from $5 \mathrm{mmol}(98 \%, 1643 \mathrm{mg}$ ) (diacetoxyiodo)benzene and $6 \mathrm{mmol}(98 \%, 405 \mathrm{mg}, 519 \mu \mathrm{L})$ cyclopropylacetylene; $317 \mathrm{mg}(0.558 \mathrm{mmol}, 11 \%$ yield, $55 \% \mathrm{E}$, $45 \% \mathrm{Z})$ beige solid. MP: $73-76{ }^{\circ} \mathrm{C}$ (dec.). ${ }^{1} \mathrm{H} \mathrm{NMR}\left(\mathrm{CD}_{2} \mathrm{Cl}_{2}\right.$, $\left.25^{\circ} \mathrm{C}, 400 \mathrm{MHz}\right), \delta(\mathrm{ppm}): 0.9-1.25\left(4 \mathrm{H}, \mathrm{m}, \mathrm{H} 2^{\prime \prime}{ }_{\mathrm{x}, \mathrm{y}}+\mathrm{H} 3^{\prime \prime}{ }_{\mathrm{x}, \mathrm{y}}\right)$; $2.24\left(1 \mathrm{H}, \mathrm{m}, \mathrm{H} 1^{\prime \prime}\right)$; 7.07 (1H, s, H1); $7.53\left(2 \mathrm{H}, \mathrm{m}, \mathrm{H}^{\prime}{ }^{\prime}+\right.$ $\left.\mathrm{H}^{\prime}\right)$, $7.69\left(1 \mathrm{H}, \mathrm{m}, \mathrm{H} 4^{\prime}\right) ; 8.00\left(2 \mathrm{H}, \mathrm{m}, \mathrm{H} 2^{\prime}+\mathrm{H}^{\prime}\right) .{ }^{13} \mathrm{C}\left\{{ }^{1} \mathrm{H}\right\}$ $\mathrm{NMR}\left(\mathrm{CD}_{2} \mathrm{Cl}_{2}, 25^{\circ} \mathrm{C}, 100 \mathrm{MHz}\right), \delta(\mathrm{ppm}): 9.3\left(\mathrm{C}^{\prime \prime}+\mathrm{C}^{\prime \prime}\right)$; 16.3 (C4"); $90.3(\mathrm{C} 1)$; $114.2\left(\mathrm{C}^{\prime}\right) ; 118.0\left(\mathrm{q},{ }^{1} J_{\mathrm{C}, \mathrm{F}}=320.0\right.$ $\left.\mathrm{Hz}, \mathrm{CF}_{3}\right) ; 120.5\left(\mathrm{q},{ }^{1} J_{\mathrm{C}, \mathrm{F}}=320.0 \mathrm{~Hz}, \mathrm{CF}_{3}\right) ; 133.0\left(\mathrm{C}^{\prime}+\mathrm{C5}^{\prime}\right)$; $133.5\left(\mathrm{C}^{\prime}\right) ; 135.9\left(\mathrm{C} 2^{\prime}+\mathrm{C}^{\prime}\right) ; 162.6(\mathrm{C} 2) .{ }^{19} \mathrm{~F}$ NMR $\left(\mathrm{CD}_{2} \mathrm{Cl}_{2}, 25^{\circ} \mathrm{C}, 376 \mathrm{MHz}\right), \delta(\mathrm{ppm}):-73.6 ;-78.9$. IR 1417, $1273,1242,1217,1182,1167,1137,1092,1048,1025,1005$, $990,930,912,856,796,761,733,677 \mathrm{~cm}^{-1}$. HRMS calculated for $\mathrm{C}_{12} \mathrm{H}_{11} \mathrm{O}_{3} \mathrm{~F}_{3} \mathrm{SI}[\mathrm{M}]^{+} 418.9426$; found 418.9429 .

7f, (E)-(2-Cyclopentyl-2-(((trifluoromethyl)sulfonyl)oxy)vinyl)(phenyl)iodonium Trifluoromethanesulfonate and (Z)-(2-Cyclopentyl-2-(((trifluoromethyl)sulfonyl)oxy)vinyl)(phenyl)iodonium Trifluoromethanesulfonate. The general procedure was followed starting from $4 \mathrm{mmol}(98 \%, 1315 \mathrm{mg}$ ) (diacetoxyiodo)benzene and $4.8 \mathrm{mmol}(90 \%, 502 \mathrm{mg}, 618 \mu \mathrm{L})$ cyclopentylacetylene; $748 \mathrm{mg}$ ( $1.25 \mathrm{mmol}, 31 \%$ yield, $80 \% \mathrm{E}$, $20 \% Z$ ) off-white solid. MP: $102-106{ }^{\circ} \mathrm{C}$ (dec.). ${ }^{1} \mathrm{H}$ NMR $\left(\mathrm{CD}_{2} \mathrm{Cl}_{2}, 25^{\circ} \mathrm{C}, 400 \mathrm{MHz}\right), \delta(\mathrm{ppm}): 1.60\left(2 \mathrm{H}, \mathrm{m}, \mathrm{H} 2^{\prime \prime}{ }_{\mathrm{y}}+\right.$ $\left.\mathrm{H}^{\prime \prime}{ }_{\mathrm{y}}\right) ; 1.66\left(2 \mathrm{H}, \mathrm{m}, \mathrm{H} 3^{\prime \prime}{ }_{\mathrm{y}}+\mathrm{H} 4^{\prime \prime}{ }_{\mathrm{y}}\right) ; 1.72\left(2 \mathrm{H}, \mathrm{m}, \mathrm{H} 3{ }^{\prime \prime}{ }_{\mathrm{x}}+\right.$ $\left.\mathrm{H} 4{ }^{\prime \prime}{ }_{\mathrm{x}}\right) ; 1.84$ (2H, m, H2 $\left.{ }_{\mathrm{x}}+\mathrm{H} 5^{\prime \prime}{ }_{\mathrm{x}}\right) ; 3.44$ (1H, m, H1 $\left.{ }^{\prime \prime}\right) ; 7.05$ $(1 \mathrm{H}, \mathrm{s}, \mathrm{H} 1) ; 7.55\left(2 \mathrm{H}, \mathrm{m}, \mathrm{H} 3^{\prime}+\mathrm{H}^{\prime}\right), 7.69\left(1 \mathrm{H}, \mathrm{m}, \mathrm{H} 4^{\prime}\right)$; 
$7.98\left(2 \mathrm{H}, \mathrm{m}, \mathrm{H} 2^{\prime}+\mathrm{H} 6^{\prime}\right) \cdot{ }^{13} \mathrm{C}\left\{{ }^{1} \mathrm{H}\right\} \operatorname{NMR}\left(\mathrm{CD}_{2} \mathrm{Cl}_{2}, 25^{\circ} \mathrm{C}, 100\right.$ $\mathrm{MHz}), \delta(\mathrm{ppm}): 26.5\left(\mathrm{C}^{\prime \prime}+\mathrm{C}^{\prime \prime}\right) ; 31.0\left(\mathrm{C}^{\prime \prime}+\mathrm{C}^{\prime \prime}\right) ; 45.1$ $\left(\mathrm{Cl}^{\prime \prime}\right)$; $89.9(\mathrm{C} 1) ; 114.6\left(\mathrm{Cl}^{\prime}\right) ; 118.0\left(\mathrm{q},{ }^{1} J_{\mathrm{C}, \mathrm{F}}=320.0 \mathrm{~Hz}\right.$, $\left.\mathrm{CF}_{3}\right) ; 120.5\left(\mathrm{q},{ }^{1} J_{\mathrm{C}, \mathrm{F}}=320.0 \mathrm{~Hz}, \mathrm{CF}_{3}\right) ; 133.0\left(\mathrm{C}^{\prime}+\mathrm{C5}^{\prime}\right)$; $133.4\left(\mathrm{C} 4^{\prime}\right) ; 135.3\left(\mathrm{C} 2^{\prime}+\mathrm{C}^{\prime}\right) ; 165.3(\mathrm{C} 2) .{ }^{19} \mathrm{~F} \mathrm{NMR}$ $\left(\mathrm{CD}_{2} \mathrm{Cl}_{2}, 25^{\circ} \mathrm{C}, 376 \mathrm{MHz}\right), \delta$ (ppm): -73.8; -78.9. IR 1426, $1277,1249,1215,1165,1135,1077,1025,992,928,856,798$, $781,751,734,679,654 \mathrm{~cm}^{-1}$. HRMS calculated for $\mathrm{C}_{14} \mathrm{H}_{15} \mathrm{O}_{3} \mathrm{~F}_{3} \mathrm{SI}[\mathrm{M}]^{+}$446.9739; found 446.9745.

7g, (E)-(2-Cyclohexyl-2-(((trifluoromethyl)sulfonyl)oxy)vinyl)(phenyl)iodonium Trifluoromethanesulfonate and (Z)-(2-Cyclohexyl-2-(((trifluoromethyl)sulfonyl)oxy) vinyl)(phenyl)iodonium Trifluoromethanesulfonate. The general procedure was followed starting from $3.75 \mathrm{mmol}$ (98\%, 1233 $\mathrm{mg}$ ) (diacetoxyiodo)benzene and $4.5 \mathrm{mmol}(98 \%, 497 \mathrm{mg}$, $600 \mu \mathrm{L})$ cyclohexylacetylene; $910 \mathrm{mg}(1.49 \mathrm{mmol}, 40 \%$ yield, 90\% E, $10 \% \mathrm{Z}$ ) off-white solid. MP: $153-156{ }^{\circ} \mathrm{C}$ (dec.). ${ }^{1} \mathrm{H}$ NMR $\left(\mathrm{CD}_{2} \mathrm{Cl}_{2}, 25{ }^{\circ} \mathrm{C}, 400 \mathrm{MHz}\right), \delta$ (ppm): $1.19(1 \mathrm{H}, \mathrm{m}$, $\left.\mathrm{H} 4{ }^{\prime \prime} \mathrm{y}\right) ; 1.30\left(2 \mathrm{H}, \mathrm{m}, \mathrm{H} 3^{\prime \prime}{ }_{\mathrm{y}}+\mathrm{H} 5^{\prime \prime}{ }_{\mathrm{y}}\right) ; 1.37\left(2 \mathrm{H}, \mathrm{m}, \mathrm{H} 2^{\prime \prime}{ }_{\mathrm{y}}+\right.$ $\left.\mathrm{H} 6{ }^{\prime \prime}\right) ; 1.59\left(2 \mathrm{H}, \mathrm{m}, \mathrm{H} 2^{\prime \prime}{ }_{\mathrm{x}}+\mathrm{H} 6^{\prime \prime}{ }_{\mathrm{x}}\right) ; 1.71$ (1H, m, H4 $\left.{ }_{\mathrm{x}}{ }_{\mathrm{x}}\right) ; 1.80$ $\left(2 \mathrm{H}, \mathrm{m}, \mathrm{H} 3^{\prime \prime}{ }_{\mathrm{x}}+\mathrm{HS}^{\prime \prime}{ }_{\mathrm{x}}\right) ; 2.98$ (1H, m, H1" $)$; 7.03 (1H, s, H1); $7.55\left(2 \mathrm{H}, \mathrm{m}, \mathrm{H} 3^{\prime}+\mathrm{H} 5^{\prime}\right), 7.70\left(1 \mathrm{H}, \mathrm{m}, \mathrm{H} 4^{\prime}\right) ; 8.00(2 \mathrm{H}, \mathrm{m}$, $\left.\mathrm{H} 2^{\prime}+\mathrm{H} 66^{\prime}\right) .{ }^{13} \mathrm{C}\left\{{ }^{1} \mathrm{H}\right\}$ NMR $\left(\mathrm{CD}_{2} \mathrm{Cl}_{2}, 25{ }^{\circ} \mathrm{C}, 100 \mathrm{MHz}\right), \delta$ (ppm): $25.5\left(\mathrm{C}^{\prime \prime}\right) ; 25.6\left(\mathrm{C}^{\prime \prime}+\mathrm{C}^{\prime \prime}\right) ; 29.5\left(\mathrm{C}^{\prime \prime}+\mathrm{C}^{\prime \prime}\right)$, $44.9\left(\mathrm{Cl}^{\prime \prime}\right) ; 89.8(\mathrm{C} 1) ; 114.8\left(\mathrm{Cl}^{\prime}\right) ; 118.0\left(\mathrm{q},{ }^{1} J_{\mathrm{C}, \mathrm{F}}=320.0\right.$ $\left.\mathrm{Hz}, \mathrm{CF}_{3}\right) ; 120.5\left(\mathrm{q},{ }^{1} J_{\mathrm{C}, \mathrm{F}}=320.0 \mathrm{~Hz}, \mathrm{CF}_{3}\right) ; 133.1\left(\mathrm{C}^{\prime}+\mathrm{C5}^{\prime}\right)$; $133.5\left(\mathrm{C}^{\prime}\right) ; 135.4\left(\mathrm{C} 2^{\prime}+\mathrm{C}^{\prime}\right) ; 166.0(\mathrm{C} 2) .{ }^{19} \mathrm{~F}$ NMR $\left(\mathrm{CD}_{2} \mathrm{Cl}_{2}, 25^{\circ} \mathrm{C}, 376 \mathrm{MHz}\right), \delta$ (ppm): -73.7; -78.9. IR 1428, 1271, 1251, 1214, 1184, 1159, 1133, 1081, 1027, 992, 977, $921,899,846,809,796,777,759,749,734,710,679,656$ $\mathrm{cm}^{-1}$. HRMS calculated for $\mathrm{C}_{15} \mathrm{H}_{17} \mathrm{O}_{3} \mathrm{~F}_{3} \mathrm{SI}[\mathrm{M}]^{+}$460.9895; found 460.9895 .

7h, (E)-Phenyl(3-phenyl-2-(((trifluoromethyl)sulfonyl)oxy)prop-1-en-1-yl)iodonium Trifluoromethanesulfonate and (Z)-Phenyl(3-phenyl-2-(((trifluoromethyl)sulfonyl)oxy)prop-1-en-1-yl)iodonium Trifluoromethanesulfonate. The general procedure was followed starting from $3.4 \mathrm{mmol}$ (98\%, $1117 \mathrm{mg}$ ) (diacetoxyiodo)benzene and $4.08 \mathrm{mmol}$ (95\%, $499 \mathrm{mg}, 531 \mu \mathrm{L}$ ) 3-phenyl-1-propyne; $1310 \mathrm{mg}(2.12$ mmol, $62 \%$ yield, $90 \% \mathrm{E}, 10 \% \mathrm{Z})$ white solid. MP: 133-139 ${ }^{\circ} \mathrm{C}$ (dec.). ${ }^{1} \mathrm{H}$ NMR $\left(\mathrm{CD}_{2} \mathrm{Cl}_{2}, 25{ }^{\circ} \mathrm{C}, 400 \mathrm{MHz}\right), \delta$ (ppm): $4.16\left(2 \mathrm{H}, \mathrm{s}, \mathrm{H} 3_{\mathrm{x}, \mathrm{y}}\right) ; 7.15(1 \mathrm{H}, \mathrm{s}, \mathrm{H} 1) ; 7.20\left(2 \mathrm{H}, \mathrm{m}, \mathrm{H} 2^{\prime \prime}+\right.$ $\left.\mathrm{H} 6^{\prime \prime}\right) ; 7.38$ (3H, m, H3" + H4" + H5" ); 7.50 (2H, m, H3' + $\left.\mathrm{H}^{\prime}\right)$, $7.69\left(1 \mathrm{H}, \mathrm{m}, \mathrm{H} 4^{\prime}\right) ; 7.87\left(2 \mathrm{H}, \mathrm{m}, \mathrm{H} 2^{\prime}+\mathrm{H}^{\prime}\right) .{ }^{13} \mathrm{C}\left\{{ }^{1} \mathrm{H}\right\}$ NMR $\left(\mathrm{CD}_{2} \mathrm{Cl}_{2}, 25{ }^{\circ} \mathrm{C}, 100 \mathrm{MHz}\right), \delta$ (ppm): 41.2 (C3); 93.5 (C1); $114.5\left(\mathrm{Cl}^{\prime}\right)$; $118.0\left(\mathrm{q},{ }^{1} \mathrm{~J}_{\mathrm{C}, \mathrm{F}}=320.0 \mathrm{~Hz}, \mathrm{CF}_{3}\right) ; 120.5$ (q, $\left.{ }^{1} J_{\mathrm{C}, \mathrm{F}}=320.0 \mathrm{~Hz}, \mathrm{CF}_{3}\right) ; 129.3\left(\mathrm{C}^{\prime \prime}\right), 129.8\left(\mathrm{C}^{\prime \prime}+\mathrm{C}^{\prime \prime}\right)$; $130.1\left(\mathrm{C}^{\prime \prime}+\mathrm{C}^{\prime \prime}\right) ; 133.1\left(\mathrm{C}^{\prime}+\mathrm{C}^{\prime}\right) ; 133.6\left(\mathrm{C}^{\prime}\right) ; 135.6$ $\left(\mathrm{C} 2^{\prime}+\mathrm{C}^{\prime}\right) ; 160.1(\mathrm{C} 2) \cdot{ }^{19} \mathrm{~F}$ NMR $\left(\mathrm{CD}_{2} \mathrm{Cl}_{2}, 25{ }^{\circ} \mathrm{C}, 376\right.$ $\mathrm{MHz}), \delta(\mathrm{ppm}):-73.5 ;-78.9$. IR 1426, 1275, 1258, 1240, $1219,1206,1184,1167,1137,1051,1025,990,895,820,781$, $736,708,693,679,654 \mathrm{~cm}^{-1}$. HRMS calculated for $\mathrm{C}_{16} \mathrm{H}_{13} \mathrm{O}_{3} \mathrm{~F}_{3} \mathrm{SI}[\mathrm{M}]^{+}$468.9582; found 468.9594.

7j, (E)-(3-Hydroxy-2-(((trifluoromethyl)sulfonyl)oxy)prop1-en-1-yl)(phenyl)iodonium Trifluoromethanesulfonate. ${ }^{6 e}$ The general procedure was followed starting from $5 \mathrm{mmol}$ (98\%, $1643 \mathrm{mg}$ ) (diacetoxyiodo)benzene and $6 \mathrm{mmol}$ (99\%, $340 \mathrm{mg}, 358 \mu \mathrm{L}$ ) 2-propyn-1-ol; $1260 \mathrm{mg}$ (2.26 mmol, 45\% yield) off-white solid. MP: $101-105{ }^{\circ} \mathrm{C}$ (dec.). ${ }^{1} \mathrm{H}$ NMR $\left(\mathrm{CD}_{2} \mathrm{Cl}_{2}, 25^{\circ} \mathrm{C}, 400 \mathrm{MHz}\right), \delta(\mathrm{ppm}): 4.51(2 \mathrm{H}, \mathrm{d}, J=2.0 \mathrm{~Hz}$, $\left.\mathrm{H} 3_{\mathrm{x}, \mathrm{y}}\right) ; 4.80(1 \mathrm{H}$, br. s, OH); $6.51(1 \mathrm{H}, \mathrm{t}, J=2.0 \mathrm{~Hz}, \mathrm{H} 1) ; 7.57$ $\left(2 \mathrm{H}, \mathrm{m}, \mathrm{H} 3^{\prime}+\mathrm{H} 5^{\prime}\right), 7.75\left(1 \mathrm{H}, \mathrm{m}, \mathrm{H} 4^{\prime}\right) ; 8.03$ (2H, m, H2' + $\left.\mathrm{H} 6{ }^{\prime}\right) .{ }^{13} \mathrm{C}\left\{{ }^{1} \mathrm{H}\right\}$ NMR $\left(\mathrm{CD}_{2} \mathrm{Cl}_{2}, 25{ }^{\circ} \mathrm{C}, 100 \mathrm{MHz}\right), \delta(\mathrm{ppm})$ :
62.0 (C3); $90.4(\mathrm{C} 1) ; 114.3\left(\mathrm{Cl}^{\prime}\right) ; 118.0\left(\mathrm{q},{ }^{1} J_{\mathrm{C}, \mathrm{F}}=320.0 \mathrm{~Hz}\right.$, $\left.\mathrm{CF}_{3}\right) ; 120.5\left(\mathrm{q}^{1} \mathrm{~J}_{\mathrm{C}, \mathrm{F}}=320.0 \mathrm{~Hz}, \mathrm{CF}_{3}\right) ; 133.0\left(\mathrm{C}^{\prime}+\mathrm{C5}^{\prime}\right)$; $134.0\left(\mathrm{C}^{\prime}\right) ; 136.4\left(\mathrm{C} 2^{\prime}+\mathrm{C}^{\prime}\right) ; 151.3(\mathrm{C} 2) .{ }^{19} \mathrm{~F}$ NMR $\left(\mathrm{CD}_{2} \mathrm{Cl}_{2}, 25{ }^{\circ} \mathrm{C}, 376 \mathrm{MHz}\right), \delta(\mathrm{ppm}):-73.1 ;-78.9$. IR 1434, $1273,1247,1219,1200,1174,1163,1139,1081,1057,1048$, 1027, 992, 973, 899, 822, 800, 764, 742, 703, 680, 665, 654, $\mathrm{cm}^{-1}$. HRMS calculated for $\mathrm{C}_{10} \mathrm{H}_{9} \mathrm{O}_{4} \mathrm{~F}_{3} \mathrm{SI}[\mathrm{M}]^{+}$408.9218; found 408.9219 .

7k, (E)-(4-Hydroxy-2-(((trifluoromethyl)sulfonyl)oxy)but1-en-1-yl)(phenyl)iodonium Trifluoromethanesulfonate and (Z)-(4-Hydroxy-2-(((trifluoromethyl)sulfonyl)oxy)but-1en-1-yl)(phenyl)iodonium Trifluoromethanesulfonate. The general procedure was followed starting from $5 \mathrm{mmol}(98 \%$, $1643 \mathrm{mg}$ ) (diacetoxyiodo)benzene and $6 \mathrm{mmol}(99 \%, 425 \mathrm{mg}$, $459 \mu \mathrm{L})$ 3-butyn-1-ol; $2043 \mathrm{mg}$ (3.57 mmol, 71\% yield, 85\% E, $15 \% \mathrm{Z})$ white solid. $\mathrm{MP}:<25{ }^{\circ} \mathrm{C}$ (dec.). ${ }^{1} \mathrm{H} \mathrm{NMR}\left(\mathrm{CD}_{2} \mathrm{Cl}_{2}, 25\right.$ $\left.{ }^{\circ} \mathrm{C}, 400 \mathrm{MHz}\right), \delta(\mathrm{ppm}): 3.00\left(2 \mathrm{H}, \mathrm{t}, J=5.6 \mathrm{~Hz}, \mathrm{H} 3_{\mathrm{x}, \mathrm{y}}\right) ; 3.94$ $\left(2 \mathrm{H}, \mathrm{t}, J=5.6 \mathrm{~Hz}, \mathrm{H} 4_{\mathrm{x}, \mathrm{y}}\right) ; 4.65(1 \mathrm{H}$, br. s, OH); $6.95(1 \mathrm{H}, \mathrm{s}$, $\mathrm{H1}) ; 7.56\left(2 \mathrm{H}, \mathrm{m}, \mathrm{H} 3^{\prime}+\mathrm{H}^{\prime}\right) ; 7.62\left(1 \mathrm{H}, \mathrm{m}, \mathrm{H} 4^{\prime}\right) ; 8.00(2 \mathrm{H}$, $\left.\mathrm{m}, \mathrm{H} 2^{\prime}+\mathrm{H} 66^{\prime}\right) \cdot{ }^{13} \mathrm{C}\left\{{ }^{1} \mathrm{H}\right\} \mathrm{NMR}\left(\mathrm{CD}_{2} \mathrm{Cl}_{2}, 25{ }^{\circ} \mathrm{C}, 100 \mathrm{MHz}\right), \delta$ (ppm): 37.9 (C3); 59.8 (C4); 96.2 (C1); 113.8 (C1'); 118.9 $\left(\mathrm{q},{ }^{1} J_{\mathrm{C}, \mathrm{F}}=320.0 \mathrm{~Hz}, \mathrm{CF}_{3}\right) ; 120.6\left(\mathrm{q},{ }^{1} J_{\mathrm{C}, \mathrm{F}}=320.0 \mathrm{~Hz}, \mathrm{CF}_{3}\right)$; $133.0\left(\mathrm{C}^{\prime}+\mathrm{C}^{\prime}\right) ; 133.7\left(\mathrm{C}^{\prime}\right) ; 135.8\left(\mathrm{C}^{\prime}+\mathrm{C}^{\prime}\right) ; 157.0$ (C2). ${ }^{19} \mathrm{~F}$ NMR $\left(\mathrm{CD}_{2} \mathrm{Cl}_{2}, 25{ }^{\circ} \mathrm{C}, 376 \mathrm{MHz}\right), \delta$ (ppm): -73.4; -78.9. IR 1419, 1284, 1236, 1217, 1173, 1135, 1089, 1027, 990, 964, 925, 887, 846, 802, 779, 762, 740, 714, 695, 679 $\mathrm{cm}^{-1}$. HRMS calculated for $\mathrm{C}_{11} \mathrm{H}_{11} \mathrm{O}_{4} \mathrm{~F}_{3} \mathrm{SI}[\mathrm{M}]^{+}$422.9375; found 422.9366 .

기, (E)-(3-Bromo-2-(((trifluoromethyl)sulfonyl)oxy)prop-1en-1-yl)(phenyl)iodonium Trifluoromethanesulfonate. The general procedure was followed starting from $5 \mathrm{mmol}(98 \%$, $1643 \mathrm{mg}$ ) (diacetoxyiodo)benzene and $6 \mathrm{mmol}$ (97\%, $736 \mathrm{mg}$, $551 \mu \mathrm{L}$ ) 3-bromo-1-propyne; $1239 \mathrm{mg}$ (1.99 mmol, $40 \%$ yield $)$ white solid. MP: $156-159{ }^{\circ} \mathrm{C}$ (dec.). ${ }^{1} \mathrm{H}$ NMR $\left(\mathrm{CD}_{2} \mathrm{Cl}_{2}, 25\right.$ $\left.{ }^{\circ} \mathrm{C}, 600 \mathrm{MHz}\right), \delta(\mathrm{ppm}): 4.53\left(2 \mathrm{H}, \mathrm{s}, \mathrm{H} 3_{\mathrm{x}, \mathrm{y}}\right) ; 7.28(1 \mathrm{H}, \mathrm{s}, \mathrm{H} 1)$; $7.55\left(2 \mathrm{H}, \mathrm{m}, \mathrm{H} 3^{\prime}+\mathrm{H} 5^{\prime}\right), 7.71\left(1 \mathrm{H}, \mathrm{m}, \mathrm{H} 4^{\prime}\right) ; 8.08(2 \mathrm{H}, \mathrm{m}$, $\left.\mathrm{H} 2^{\prime}+\mathrm{H} 6{ }^{\prime}\right) \cdot{ }^{13} \mathrm{C}\left\{{ }^{1} \mathrm{H}\right\} \mathrm{NMR}\left(\mathrm{CD}_{2} \mathrm{Cl}_{2}, 25{ }^{\circ} \mathrm{C}, 150 \mathrm{MHz}\right), \delta$ $(\mathrm{ppm}): 27.9$ (C3); $95.5(\mathrm{C} 1) ; 114.1\left(\mathrm{Cl}^{\prime}\right) ; 118.0\left(\mathrm{q},{ }^{1} J_{\mathrm{C}, \mathrm{F}}=\right.$ $\left.320.0 \mathrm{~Hz}, \mathrm{CF}_{3}\right) ; 120.5\left(\mathrm{q},{ }^{1} J_{\mathrm{C}, \mathrm{F}}=320.0 \mathrm{~Hz}, \mathrm{CF}_{3}\right) ; 133.1\left(\mathrm{C}^{\prime}+\right.$ $\left.\mathrm{C} 5^{\prime}\right)$; $133.7\left(\mathrm{C}^{\prime}\right)$; $136.1\left(\mathrm{C}^{\prime}+\mathrm{C}^{\prime}\right) ; 156.4(\mathrm{C} 2) .{ }^{19} \mathrm{~F}$ NMR $\left(\mathrm{CD}_{2} \mathrm{Cl}_{2}, 25{ }^{\circ} \mathrm{C}, 565 \mathrm{MHz}\right), \delta(\mathrm{ppm}):-73.0 ;-78.8$. IR 1428, $1275,1264,1243,1234,1217,1178,1158,1135,1066,1022$, $988,908,822,785,757,736,708,679,665,654 \mathrm{~cm}^{-1}$. HRMS calculated for $\mathrm{C}_{10} \mathrm{H}_{8} \mathrm{O}_{3} \mathrm{~F}_{3} \mathrm{SBrI}[\mathrm{M}]^{+}$470.8374; found 470.8373. XRD: see in SI.

$7 m$, (E)-(5-Chloro-2-(((trifluoromethyl)sulfonyl)oxy)pent1-en-1-yl)(phenyl)iodonium Trifluoromethanesulfonate. The general procedure was followed starting from $5 \mathrm{mmol}$ (98\%, $1643 \mathrm{mg}$ ) (diacetoxyiodo)benzene and $6 \mathrm{mmol}(96 \%$, $641 \mathrm{mg}, 657 \mu \mathrm{L})$ 5-chloro-1-pentyne; $1991 \mathrm{mg}(3.29 \mathrm{mmol}$, $66 \%$ yield) white solid. MP: $84-89{ }^{\circ} \mathrm{C}$ (dec.). ${ }^{1} \mathrm{H}$ NMR $\left(\mathrm{CD}_{2} \mathrm{Cl}_{2}, 25^{\circ} \mathrm{C}, 400 \mathrm{MHz}\right), \delta(\mathrm{ppm}): 2.08\left(2 \mathrm{H}, \mathrm{m}, \mathrm{H} 4_{\mathrm{x}, \mathrm{y}}\right)$; $3.04\left(2 \mathrm{H}, \mathrm{t}, J=7.4 \mathrm{~Hz}, \mathrm{H} 3_{\mathrm{x}, \mathrm{y}}\right) ; 3.61\left(2 \mathrm{H}, \mathrm{t}, J=6.1 \mathrm{~Hz}, \mathrm{H} 5_{\mathrm{x}, \mathrm{y}}\right)$; $7.17(1 \mathrm{H}, \mathrm{s}, \mathrm{H} 1) ; 7.56\left(2 \mathrm{H}, \mathrm{m}, \mathrm{H}^{\prime}+\mathrm{H}^{\prime}\right), 7.70(1 \mathrm{H}, \mathrm{m}$, $\left.\mathrm{H}^{\prime}\right) ; 8.03\left(2 \mathrm{H}, \mathrm{m}, \mathrm{H} 2^{\prime}+\mathrm{H} 6^{\prime}\right) .{ }^{13} \mathrm{C}\left\{{ }^{1} \mathrm{H}\right\} \mathrm{NMR}\left(\mathrm{CD}_{2} \mathrm{Cl}_{2}, 25\right.$ ${ }^{\circ} \mathrm{C}, 100 \mathrm{MHz}$ ), $\delta$ (ppm): 29.0 (C4); 32.7 (C3); 43.9 (C5); $94.2(\mathrm{C} 1) ; 114.3\left(\mathrm{Cl}^{\prime}\right) ; 118.0\left(\mathrm{q},{ }^{1} J_{\mathrm{C}, \mathrm{F}}=320.0 \mathrm{~Hz}, \mathrm{CF}_{3}\right)$; $120.5\left(\mathrm{q},{ }^{1} J_{\mathrm{C}, \mathrm{F}}=320.0 \mathrm{~Hz}, \mathrm{CF}_{3}\right) ; 133.1\left(\mathrm{C}^{\prime}+\mathrm{C}^{\prime}\right) ; 133.6$ $\left(\mathrm{C} 4^{\prime}\right) ; 135.6\left(\mathrm{C} 2^{\prime}+\mathrm{C}^{\prime}\right) ; 161.3(\mathrm{C} 2) .{ }^{19} \mathrm{~F} \mathrm{NMR}\left(\mathrm{CD}_{2} \mathrm{Cl}_{2}, 25\right.$ $\left.{ }^{\circ} \mathrm{C}, 376 \mathrm{MHz}\right), \delta$ (ppm): -73.5 ; -78.9. IR 1435, 1428, 1279, $1266,1242,1227,1210,1186,1171,1161,1139,1057,1025$, $992,975,900,861,816,736,686,680 \mathrm{~cm}^{-1}$. HRMS calculated for $\mathrm{C}_{12} \mathrm{H}_{12} \mathrm{O}_{3} \mathrm{~F}_{3}$ SClI $[\mathrm{M}]^{+}$454.9193; found 454.9200 . 
7n, (E)-(5-Cyano-2-(((trifluoromethyl)sulfonyl)oxy)pent-1en-1-yl)(phenyl)iodonium Trifluoromethanesulfonate and (Z)-(5-Cyano-2-(((trifluoromethyl)sulfonyl)oxy)pent-1-en-1yl)(phenyl)iodonium Trifluoromethanesulfonate. The general procedure was followed starting from $5 \mathrm{mmol}$ (98\%, 1643 $\mathrm{mg}$ ) (diacetoxyiodo)benzene and $6 \mathrm{mmol}(98 \%, 570 \mathrm{mg}, 641$ $\mu \mathrm{L})$ 5-cyano-1-pentyne; $2491 \mathrm{mg}(4.18 \mathrm{mmol}, 84 \%$ yield, $95 \%$ E, 5\% Z) light yellow solid. MP: $70-75{ }^{\circ} \mathrm{C}$ (dec.). ${ }^{1} \mathrm{H}$ NMR $\left(\mathrm{CD}_{2} \mathrm{Cl}_{2}, 25^{\circ} \mathrm{C}, 400 \mathrm{MHz}\right), \delta(\mathrm{ppm}): 2.00\left(2 \mathrm{H}, \mathrm{m}, \mathrm{H} 4_{\mathrm{x}, \mathrm{Y}}\right)$ $2.52\left(2 \mathrm{H}, \mathrm{t}, J=6.5 \mathrm{~Hz}, \mathrm{H5}_{\mathrm{x}, \mathrm{y}}\right) ; 3.03\left(2 \mathrm{H}, \mathrm{t}, J=7.0 \mathrm{~Hz}, \mathrm{H}_{\mathrm{x}, \mathrm{y}}\right)$; $7.20(1 \mathrm{H}, \mathrm{s}, \mathrm{H1}) ; 7.56\left(2 \mathrm{H}, \mathrm{m}, \mathrm{H} 3^{\prime}+\mathrm{H}^{\prime}\right), 7.73(1 \mathrm{H}, \mathrm{m}$ $\left.\mathrm{H} 4^{\prime}\right) ; 8.04\left(2 \mathrm{H}, \mathrm{m}, \mathrm{H} 2^{\prime}+\mathrm{H}^{\prime}\right) \cdot{ }^{13} \mathrm{C}\left\{{ }^{1} \mathrm{H}\right\}$ NMR $\left(\mathrm{CD}_{2} \mathrm{Cl}_{2}, 25\right.$ ${ }^{\circ} \mathrm{C}, 100 \mathrm{MHz}$ ), $\delta$ (ppm): 16.9 (C5); 22.4 (C4); 34.1 (C3); $94.5(\mathrm{C} 1) ; 114.1\left(\mathrm{Cl}^{\prime}\right)$; $117.3(\mathrm{CN}) ; 118.0\left(\mathrm{q},{ }^{1} J_{\mathrm{C}, \mathrm{F}}=320.0\right.$ $\left.\mathrm{Hz}, \mathrm{CF}_{3}\right) ; 120.5\left(\mathrm{q},{ }^{1} J_{\mathrm{C}, \mathrm{F}}=320.0 \mathrm{~Hz}, \mathrm{CF}_{3}\right) ; 133.2\left(\mathrm{C}^{\prime}+\mathrm{C}^{\prime}\right)$; $133.7\left(\mathrm{C}^{\prime}\right) ; 135.6\left(\mathrm{C} 2^{\prime}+\mathrm{C}^{\prime}\right) ; 160.5(\mathrm{C} 2) .{ }^{19} \mathrm{~F}$ NMR $\left(\mathrm{CD}_{2} \mathrm{Cl}_{2}, 25^{\circ} \mathrm{C}, 376 \mathrm{MHz}\right), \delta$ (ppm): $-73.3 ;-78.8$. IR 1428, $1273,1240,1225,1214,1193,1174,1163,1135,1063,1023$, 992, 979, 910, 904, 876, 815, 738, 716, $679 \mathrm{~cm}^{-1}$. HRMS calculated for $\mathrm{C}_{13} \mathrm{H}_{12} \mathrm{NO}_{3} \mathrm{~F}_{3} \mathrm{SI}[\mathrm{M}]^{+}$445.9535; found 445.9536 .

\section{ASSOCIATED CONTENT}

\section{S Supporting Information}

The Supporting Information is available free of charge on the ACS Publications website at DOI: 10.1021/acsomega.9b00728.

CCDC numbers 1879587-1879592 for structures $7 \mathbf{a}$, $7 \mathrm{~b}, 7 \mathrm{~d}, 7 \mathbf{l}, \mathbf{6 a}$, and $\mathbf{6 c}$, respectively (PDF)

Crystal data, data collection and structure refinement details (CIF)

\section{AUTHOR INFORMATION}

\section{Corresponding Authors}

*E-mail: tothb@zng.elte.hu. Phone: +36 13722500 \#1608 (B.L.T.).

*E-mail: novakz@elte.hu (Z.N.).

\section{ORCID}

András Stirling: 0000-0002-1696-7932

Zoltán Novák: 0000-0001-5525-3070

\section{Notes}

The authors declare no competing financial interest.

\section{ACKNOWLEDGMENTS}

This project was supported by the ÚNKP-17-3 New National Excellence Program of the Ministry of Human Capacities. The authors acknowledge the support of the National Research, Development and Innovation Office (Grant Nos. K125120 and K116034), and the Janos Bolyai Research Scholarship of the Hungarian Academy of Sciences. The research was also supported by the EU and cofinanced by the European Regional Development Fund under the projects GINOP2.3.2-15-2016-00008 and GINOP-2.3.3-15-2016-00004. This work was completed in the ELTE Institutional Excellence Program (1783-3/2018/FEKUTSRAT) supported by the Hungarian Ministry of Human Capacities

\section{REFERENCES}

(1) (a) Bouma, M. J.; Olofsson, B. General One-Pot Synthesis of Alkynyliodonium Salts and Alkynyl Benziodoxolones from Aryl Iodides. Chem. Eur. J. 2012, 18, 14242-14245. (b) Zhdankin, V. V. Hypervalent Iodine Chemistry: Preparation, Structure, and Synthetic
Applications of Polyvalent Iodine Compounds; Wiley: Chichester, U.K., 2013. (c) Olofsson, B. Arylation with Diaryliodonium Salts. Hypervalent Iodine Chemistry; Topics in Current Chemistry; Springer: Cham, 2015; Vol. 373, pp 135-166. (d) Aradi, K.; Tóth, B.; Tolnai, G.; Novák, Z. Diaryliodonium Salts in Organic Syntheses: A Useful Compound Class for Novel Arylation Strategies. Synlett 2016, 27, 1456-1485. (e) Yoshimura, A.; Zhdankin, V. V. Advances in Synthetic Applications of Hypervalent Iodine Compounds. Chem. Rev. 2016, 116, 3328-3435.

(2) (a) Pirkuliev, N. S.; Brel, V. K.; Zefirov, N. S. Alkenyliodonium salts. Russ. Chem. Rev. 2000, 69, 105-120. (b) Ochiai, M. J. Nucleophilic vinylic substitutions of $\lambda^{3}$-vinyliodanes. J. Orgnomet. Chem. 2000, 611, 494-508. (c) Okuyama, T.; Fujita, M. Reactions of Cyclohexenyliodonium Salts. Russ. J. Org. Chem. 2005, 41, 12451253. (d) Chatterjee, N.; Goswami, A. Diverse transformations of Boronic Compounds Promoted by Hypervalent Organoiodines(III): Unique Combined Reactivity of Two Electrophilic Compounds. Adv. Synth. Catal. 2017, 359, 358-371.

(3) (a) Koser, G. F.; Rebrovic, L.; Wettach, R. H. Functionalization of alkenes and alkynes with [hydroxy(tosyloxy)iodo]benzene. Bis(tosyloxy)alkanes, vinylaryliodonium tosylates, and alkynylaryliodonium tosylates. J. Org. Chem. 1981, 46, 4324-4326. (b) Ochiai, M.; Takaoka, Y.; Nagao, Y. Hypervalent alkenyliodonium tetrafluoroborates. Evidence for generation of alkylidenecarbenes via base-induced $\alpha$-elimination. J. Am. Chem. Soc. 1988, 110, 6565-6566. (c) Ochiai, M.; Oshima, K.; Masaki, Y. Stereoselective synthesis of highly labile (Z)- $\beta$-alkylvinyl(phenyl)iodonium perchlorates. J. Chem. Soc. Chem. Commun. 1991, 0, 869-870. (d) Stang, P.; Ullmannä, J. Synthesis of Ethenyl(phenyl)iodonium Triflate, $\left[\mathrm{H}_{2} \mathrm{C}=\mathrm{CHIPh}\right]\left[\mathrm{OSO}_{2} \mathrm{CF}_{3}\right]$, and Its Application as a Parent Vinyl Cation Equivalent. Angew. Chem., Int. Ed. 1991, 30, 1469-1470. (e) Williamson, B. L.; Stang, P. J.; Arif, A. M. Preparation, molecular structure, and Diels-Alder cycloaddition chemistry of $\beta$-functionalized alkynyl(phenyl)iodonium salts. J. Am. Chem. Soc. 1993, 115, 2590-2597. (f) Murch, P.; Arif, A. M.; Stang, P. J. Regiochemistry of Diels-Alder Reactions of Diverse $\beta$ Functionalized Alkynyliodonium Salts with Unsymmetrical Dienes. J. Org. Chem. 1997, 62, 5959-5965. (g) Yoshida, M.; Hara, S. Stereoselective Synthesis of (Z)-2-Fluoro-1-alkenyl(phenyl)iodonium Tetrafluoroborates. Org. Lett. 2003, 5, 573-574. (h) Thielges, S.; Bisseret, P.; Eustache, J. Copper-Mediated Cross-Coupling of $\mathrm{H}$ Phosphonates with Vinyliodonium Salts: A Novel Very Mild Synthesis of 2-Arylvinylphosphonates. Org. Lett. 2005, 7, 681-684. (i) Ochiai, M.; Hirobe, M.; Yoshimura, A.; Nishi, Y.; Miyamoto, K.; Shiro, M. Internal Delivery of Soft Chlorine and Bromine Atoms: Stereoselective Synthesis of (E)- $\beta$-Halogenovinyl(aryl)- $\lambda 3$-iodanes through Domino $\lambda^{3}$-Iodanation-1,4-Halogen Shift-Fluorination of Alkynes. Org. Lett. 2007, 9, 3335-3338. (j) Shimizu, M.; Takeda, Y.; Hiyama, T. Preparation, Structure, and Diels-Alder Reaction of Phenyl(trifluoromethanesulfonate) (3,3,3-trifluoropropynyl)- $\lambda 3$-iodane. Chem. Lett. 2008, 37, 1304-1305. (k) Shah, A.-u.-H. A.; Khan, Z. A.; Choudhary, N.; Lohölter, C.; Schäfer, S.; Marie, G. P. L.; Farooq, U.; Witulski, B.; Wirth, T. Iodoxolone-Based Hypervalent Iodine Reagents. Org. Lett. 2009, 11, 3578-3581. (1) Justik, M. W.; Kristufek, S. L.; Protasiewicz, J. D.; Deligonul, N. Stereoselective Synthesis and X-ray Structures of Alkenyliodonium Salts with a Pyridine N-Oxide Moiety. Synthesis 2010, 2010, 2345-2347. (m) Merritt, E. A.; Olofsson, B. Synthesis of a Range of Iodine(III) Compounds Directly from Iodoarenes. Eur. J. Org. Chem. 2011, 3690-3694. (n) Hyatt, I. F. D.; Croatt, M. P. Reactions of Hypervalent Iodonium Alkynyl Triflates with Azides: Generation of Cyanocarbenes. Angew. Chem., Int. Ed. 2012, 51, 7511-7514. (o) Zawia, E.; Moran, W. Aqueous DMSO Mediated Conversion of (2-(Arylsulfonyl)vinyl)iodonium Salts to Aldehydes and Vinyl Chlorides. Molecules 2016, 21, 1073. (p) Wu, J.; Deng, X.; Hirao, H.; Yoshikai, N. Pd-Catalyzed Conversion of Alkynyl- $\lambda 3$-iodanes to Alkenyl- $\lambda 3$-iodanes via Stereoselective 1,2-Iodine(III) Shift/1,1Hydrocarboxylation. J. Am. Chem. Soc. 2016, 138, 9105-9108. (q) Stridfeldt, E.; Seemann, A.; Bouma, M. J.; Dey, C.; Ertan, A.; Olofsson, B. Synthesis, Characterization and Unusual Reactivity of 
Vinylbenziodoxolones-Novel Hypervalent Iodine Reagents. Chem. Eur. J. 2016, 22, 16066-16070. (r) Kitamura, T.; Mizuno, S.; Muta, $\mathrm{K}$.; Oyamada, J. Synthesis of $\beta$-Fluorovinyliodonium Salts by the Reaction of Alkynes with Hypervalent Iodine/HF Reagents. J. Org. Chem. 2018, 83, 2773-2778.

(4) (a) Lodaya, J. S.; Koser, G. F. Alkynyliodonium salts as alkynylating reagents: direct conversion of alkynylphenyliodonium tosylates to dialkyl alkynylphosphonates with trialkyl phosphites. J. Org. Chem. 1990, 55, 1513-1516. (b) Moriarty, R. M.; Epa, W. R.; Awasthi, A. K. Palladium-catalyzed coupling of alkenyl iodonium salts with olefins: a mild and stereoselective Heck-type reaction using hypervalent iodine. J. Am. Chem. Soc. 1991, 113, 6315-6317. (c) Stang, P. J.; Schwarz, A.; Blume, T.; Zhdankin, V. V. Reactions of bicycloalkenyldiiodonium salts with nucleophiles. Tetrahedron Lett. 1992, 33, 6759-6762. (d) Ochiai, M.; Kitagawa, Y.; Yamamoto, S. Generation and Reaction of Monocarbonyliodonium Ylides: Ester Exchange of $(Z)$-( $\beta$-Acetoxyvinyl)iodonium Salts with Lithium Ethoxide and Synthesis of $\alpha, \beta$-Epoxy Ketones. J. Am. Chem. Soc. 1997, 119, 11598-11604. (e) Hara, S.; Yoshida, M.; Fukuhara, T.; Yoneda, N. Stereo- and regio-selective addition of iodotoluene difluoride to alk-1-ynes. Selective synthesis of 2-fluoro-1-iodoalk-1enes. Chem. Commun. 1998, 965-966. (f) Hara, S.; Yamamoto, K.; Yoshida, M.; Fukuhara, T.; Yoneda, N. Stereoselective synthesis of (E)- $\beta$-fluoro- $\alpha, \beta$-unsaturated esters by carbonylation of $(E)$-2-fluoro1-iodo-1-alkenyliodonium salts. Tetrahedron Lett. 1999, 40, 78157818. (g) Okuyama, T. Solvolysis of Vinyl Iodonium Salts. New Insights into Vinyl Cation Intermediates. Acc. Chem. Res. 2002, 35, 12-18. (h) Fujita, M.; Ihara, K.; Kim, W. H.; Okuyama, T. Generation of Cycloheptyne during the Solvolysis of Cyclohexylidenemethyliodonium Salt in the Presence of Base. Bull. Chem. Soc. Jpn. 2003, 76, 1849-1855. (i) Aggarwal, V. K.; Olofsson, B. Enantioselective $\alpha$-Arylation of Cyclohexanones with Diaryl Iodonium Salts: Application to the Synthesis of (-)-Epibatidine. Angew. Chem., Int. Ed. 2005, 44, 5516-5519. (j) Yoshida, M.; Komata, A.; Hara, S. Stereoselective synthesis of fluoroalkenes via (Z)-2fluoroalkenyliodonium salts. Tetrahedron 2006, 62, 8636-8645. (k) Miyamoto, K.; Suzuki, M.; Suefuji, T.; Ochiai, M. In Situ Generation Technology of $\beta$-Butoxycarbonyliodonium Ylide: A Hypervalent Analogue of the Darzens Reagent. Eur. J. Org. Chem. 2013, 3662-3666. (1) Guo, T.; Jiang, Q.; Yu, Z. Copper-Catalyzed Ring-Expansion/Thiolactonization via Azidation of Internal Olefinic $\mathrm{C}-\mathrm{H}$ Bond under Mild Conditions. Adv. Synth. Catal. 2016, 358, 3450-3457. (m) Zhang, L.; Oestreich, M. Copper-Catalyzed CrossCoupling of Vinyliodonium Salts and Zinc-Based Silicon Nucleophiles. Org. Lett. 2018, 20, 8061-8063.

(5) (a) Skucas, E.; MacMillan, D. W. C. Enantioselective $\alpha$ Vinylation of Aldehydes via the Synergistic Combination of Copper and Amine Catalysis. J. Am. Chem. Soc. 2012, 134, 9090-9093. (b) Suero, M. G.; Bayle, E. D.; Collins, B. S. L.; Gaunt, M. J. CopperCatalyzed Electrophilic Carbofunctionalization of Alkynes to Highly Functionalized Tetrasubstituted Alkenes. J. Am. Chem. Soc. 2013, 135, 5332-5335. (c) Holt, D.; Gaunt, M. J. Copper-Catalyzed OxyAlkenylation of Homoallylic Alcohols to Generate Functionalsyn-1,3Diol Derivatives. Angew. Chem., Int. Ed. 2015, 54, 7857-7861. (d) Pankajakshan, S.; Ang, W. L.; Sreejith, S.; Stuparu, M. C.; Loh, T.P. Aerobic Copper Catalysis for Tandem Oxy-N-alkenylation of $[1,2,3]$ Triazolo $[1,5-a]$ pyridines. Adv. Synth. Catal. 2016, 358, 30343038. (e) Liu, C.; Wang, Q. Arylation, Vinylation, and Alkynylation of Electron-Deficient (Hetero)arenes Using Iodonium Salts. Org. Lett. 2016, 18, 5118-5121. (f) Guo, J.; Lin, L.; Liu, Y.; Li, X.; Liu, X.; Feng, X. Nickel(II)-Catalyzed Enantioselective $\alpha$-Vinylation of $\beta$-Keto Amides/Esters with Hypervalent Iodine Salts. Org. Lett. 2016, 18, 5540-5543. (g) Hinkle, R. J.; Leri, A. C.; David, G. A.; Erwin, W. M. Addition of Benzylzinc Halides to Alkenyl(phenyl)iodonium Triflates: Stereoselective Synthesis of Trisubstituted Alkenes. Org. Lett. 2000, 2, 1521-1523. (h) Sheng, J.; Wang, Y.; Su, X.; He, R.; Chen, C. CopperCatalyzed $[2+2+2]$ Modular Synthesis of Multisubstituted Pyridines: Alkenylation of Nitriles with Vinyliodonium Salts. Angew. Chem., Int. Ed. 2017, 56, 4824-4828. (i) Teskey, C. J.; Sohel, S. M.
A.; Bunting, D. L.; Modha, S. G.; Greaney, M. F. Domino N -/C -Arylation via In Situ Generation of a Directing Group: AtomEfficient Arylation Using Diaryliodonium Salts. Angew. Chem., Int. Ed. 2017, 56, 5263-5266. (j) Boelke, A.; Caspers, L. D.; Nachtsheim, B. J. $\mathrm{NH}_{2}$-Directed $\mathrm{C}-\mathrm{H}$ Alkenylation of 2-Vinylanilines with Vinylbenziodoxolones. Org. Lett. 2017, 19, 5344-5347. (k) Rajkiewicz, A. A.; Kalek, M. N-Heterocyclic Carbene-Catalyzed Olefination of Aldehydes with Vinyliodonium Salts To Generate $\alpha, \beta$-Unsaturated Ketones. Org. Lett. 2018, 20, 1906-1909. (1) Liu, C.; Wang, Q. Alkenylation of $\mathrm{C}\left(\mathrm{sp}^{3}\right)-\mathrm{H}$ Bonds by Zincation/Copper-Catalyzed Cross-Coupling with Iodonium Salts. Angew. Chem., Int. Ed. 2018, 57, $4727-4731$

(6) (a) Kitamura, T.; Furuki, R.; Taniguchi, H.; Stang, P. J. Stereoselective anti-addition of $\mathrm{PhIO} \cdot \mathrm{TfOH}$ to terminal alkynes. Preparation of E-( $\beta$-trifluoromethanesulfonyloxyvinyl)-iodonium triflates. Tetrahedron Lett. 1990, 31, 703-704. (b) Kitamura, T.; Furuki, R.; Taniguchi, H.; Stang, P. J. Activation of lodosylbenzene with One Equivalent of Triflic (Trifluoromethanesulphonic) Anhydride. Novel Preparation of ( $\mathrm{p}$-Phenylene)bisiodonium Triflates. Mendeleev Commun. 1991, 1, 148-149. (c) Kitamura, T.; Furuki, R; Zheng, L.; Nagata, K.; Fukuoka, T.; Fujiwara, Y.; Taniguchi, H. Alkenyl- and Alkynyl-Substituted ( $\mathrm{p}$-Phenylene)bisiodonium Ditriflates by Reactions of a (p-Phenylene)bisiodine(III) Reagent with Alkynes and 1Trimethylsilyl-1-alkynes. Bull. Chem. Soc. Jpn. 1995, 68, 3637-3641. (d) Zhdankin, V. V.; Kuehl, C. J.; Krasutsky, A. P.; Bolz, J. T.; Simonsen, A. J. 1-(Organosulfonyloxy)-3(1H)-1,2-benziodoxoles: Preparation and Reactions with Alkynyltrimethylsilanes. J. Org. Chem. 1996, 61, 6547-6551. (e) Kasumov, T. M.; Pirguliyev, N. S.; Brel, V. K.; Grishin, Y. K.; Zefirov, N. S.; Stang, P. J. New one-pot method for the stereoselective synthesis of $(E)-[\beta$-(trifluoromethylsulfonyloxy)-alkenyl] (Aryl) iodonium triflates. Tetrahedron 1997, 53, 13139-13148. (f) Kitamura, T.; Kotani, M.; Fujiwara, Y. An Alternative Synthesis of Alkynyl(phenyl)iodonium Triflates Using (Diacetoxyiodo)benzene and Alkynylsilanes. Synthesis 1998, 10, 1416-1418. (g) Pirguliyev, N. S.; Brel, V. K.; Kasumov, T. M.; Grishin, Y. K.; Zefirov, N. S.; Stang, P. J. Xenon Fluorotriflate: An Efficient Reagent for the Synthesis of (p-Phenylene)bisiodonium Salts. Synthesis 1999, 8, 1297-1299.

(7) (a) Kitamura, T.; Kotani, M.; Fujiwara, Y. An efficient ligand exchange reaction of $\beta$-(trifyloxy)vinyliodonium triflates with aryllithium reagents leading to diaryliodonium triflates. Tetrahedron Lett. 1996, 37, 3721-3722. (b) Pirguliyev, N. S.; Brel, V. K.; Akhmedov, N. G.; Zefirov, N. S. An Efficient Ligand Exchange Reaction of $(E)-[(\beta$-(Trifluoromethanesulfonyloxy)ethenyl] (phenyl)iodonium Triflates with Aryl- and Alkynyllithium Reagents Leading to Diaryl- and Alkynyliodonium Triflates. Synthesis 2000, 2000, 81-83. (c) Jalalian, N.; Olofsson, B. Design and asymmetric synthesis of chiral diaryliodonium salts. Tetrahedron 2010, 66, 5793-5800.

(8) Pirguliyev, N.; Brel, V.; Zefirov, N.; Stang, P. Stereoselective synthesis of conjugated alkenynes via palladium-catalyzed coupling of alkenyl iodonium salts with terminal alkynes. Tetrahedron 1999, 55, 12377-12386.

(9) Saito, A.; Taniguchi, A.; Kambara, Y.; Hanzawa, Y. Metal-Free [2 $+2+1]$ Annulation of Alkynes, Nitriles, and Oxygen Atoms: Iodine(III)-Mediated Synthesis of Highly Substituted Oxazoles. Org. Lett. 2013, 15, 2672-2675.

(10) Kitamura, T.; Furuki, R.; Taniguchi, H.; Stang, P. J. Electrophilic additions of iodosylbenzene activated by trifluoromethanesulfonic acid, [PhIO-TfOH], to alkynes. Tetrahedron 1992, 48, $7149-7156$.

(11) Similar conditions: (a) Zefirov, N. S.; Zhdankin, V. V.; Dankov, Y.; Sorokin, V. D.; Semerikov, V. N.; Kozmin, A. S.; Caple, R.; Berglund, B. A. Novel reagents containing hypervalent iodine and their use for electrophilic additions to olefins. Tetrahedron Lett. 1986, 27, 3971-3974. (b) Hembre, R. T.; Scott, C. P.; Norton, J. R. Conversion of olefins to ditriflates by $\mu$-oxobis[(trifluoromethanesulfonato)(phenyl)iodine]. J. Org. Chem. 1987, 52, 3650-3654. (c) Kitamura, T.; Furuki, R.; Nagata, K.; Taniguchi, H.; Stang, P. J. Preparation of (p-phenylene)bis(aryliodonium) 
ditriflates and their double substitution by some nucleophiles. J. Org. Chem. 1992, 57, 6810-6814. (d) Kitamura, T.; Matsuyuki, J.-i.; Taniguchi, H. Improved Preparation of Diaryliodonium Triflates. Synthesis 1994, 1994, 147-148. (e) Singh, F. V.; Rehbein, J.; Wirth, T. Facile Oxidative Rearrangements Using Hypervalent Iodine Reagents. ChemistryOpen 2012, 1, 245-250. (f) Farid, U.; Wirth, T. Highly Stereoselective Metal-Free Oxyaminations Using Chiral Hypervalent Iodine Reagents. Angew. Chem., Int. Ed. 2012, 51, 3462-3465. (g) Yoshimura, A.; Nguyen, K. C.; Klasen, S. C.; Saito, A.; Nemykin, V. N.; Zhdankin, V. V. Preparation, structure, and versatile reactivity of pseudocyclic benziodoxole triflate, new hypervalent iodine reagent. Chem. Commun. 2015, 51, 7835-7838.

(h) Kiyokawa, K.; Takemoto, K.; Yahata, S.; Kojima, T.; Minakata, S. Oxidative Cyclization of $\beta, \gamma$-Unsaturated Carboxylic Acids Using Hypervalent Iodine Reagents: An Efficient Synthesis of 4-Substituted Furan-2-ones. Synthesis 2017, 49, 2907-2912.

(12) See details in Supporting Information. 